\title{
Neuro-Developmental Engineering: towards early diagnosis of neuro-developmental disorders
}

\author{
${ }^{1}$ Domenico Campolo, Fabrizio Taffoni, Giuseppina Schiavone, \\ Domenico Formica, Eugenio Guglielmelli and Flavio Keller \\ Università Campus Bio-Medico \\ 00128 Roma - Italy \\ ${ }^{1}$ School of Mechanical \& Aerospace Engineering \\ Nanyang Technological University \\ 639798 Singapore
}

\section{Introduction}

Neuro-Developmental Engineering (NDE) is a new and emerging interdisciplinary research area at the intersection of developmental neuroscience and bioengineering aiming at providing new methods and tools for: $i$ ) understanding neuro-biological mechanisms of human brain development; ii) quantitative analysis and modeling of human behavior during neurodevelopment; iii) assessment of neuro-developmental milestones achieved by humans from birth onwards.

Main application fields of NDE are:

- New clinical protocols and standards for early diagnosis, functional evaluation and therapeutic treatments of neuro- developmental disorders;

- New generations of educational, interactive toys which can provide adequate stimuli and guidance for supporting the physiological neuro-development process

This technology is expected to be also useful in the long term for developing new tools, e.g. toys, which can sustain, in ecological scenarios, the regular development of motor and cognitive abilities of the child, based on a rigorous scientific approach.

The long term goal is establishing standards against which development of infants at risk for neuro-developmental disorders, particularly autism, can be measured, with the aim of detecting early signs of disturbed development.

\subsection{Sensori-Motor Integration Deficits in Neurodevelopmental Disorders}

Neurodevelopmental disorders such as ASD, ADHD, Tourette syndrome and others are characterized by a genetic basis. In this case behavioral analysis, or behavioral phenotyping, will be instrumental for the analysis of the roles of genes in behavior (Gerlai 2002).

Autism is a behavioral disorder, with onset in childhood, which is characterized by deficits in three basic domains: social interaction, language and communication, and pattern of interests. There is no doubt that autism has a strong genetic component, and that biological disease mechanisms leading to autism are already active during foetal development and/or infancy, 
as demonstrated, for example, by the abnormal pattern of brain growth during late foetal and early postnatal life (see (Keller and Persico 2003), for a review). Autism is usually diagnosed at the age of 3 years, in many cases after a period of seemingly normal neurological and behavioral development. The diagnosis of autism is purely clinical, there are no laboratory tests to confirm or disprove the diagnosis. It has been recognized that, although typical autism is not associated with major neurological deficits, autism has characteristic manifestations in the perceptual and motor domains.

Deficits in the perceptual domain include altered processing and recognition of socially relevant information from peopleŠs faces (see (Grelotti et al. 2003), for a review), deficits in perception of motion cues (Milne et al. 2002), (Spencer et al. 2000), (Bertone et al. 2003), (Takerae et al. 2004), difficulty in disengaging attention (Landry and Bryson 2004) and alterations of auditory processing (Courchesne et al. 1984), (Boddaert et al. 2004). Studies based on analysis of home-made movies suggest that an impairment of spontaneous attention toward social stimuli is present already at 20 months (Swettenham et al. 1998), and possibly also as early as during the first 6 months of life (Maestro et al. 2002). Furthermore, an autism-like syndrome is frequently observed in congenitally blind children (Hobson and Bishop 2003). Taken together, these observations suggest that at least some individuals with autism are characterized by an early deficit of 'low-level' perceptual processing, which jeopardizes their ability to develop higher-level capacities, such as language and interpersonal skills.

Motor impairments in autism include deficits in postural reflexes (Minshew et al. 2004), (Schmitz et al. 2003), (Molloy et al. 2003), repetitive, stereotyped movements and awkward patterns of object manipulation, lack of purposeful exploratory movements (see e.g. (Pierce and Courchesne 2001)), gaze abnormalities (Sweeney et al. 2004), unusual gait pattern (Hallett et al. 1993), and alterations of movement planning and execution, which express themselves as 'hyper- dexterity' (Rinehart et al. 2001), (Mari et al. 2003). Motor abnormalities may be observed retrospectively in infants who later develop the autistic syndrome, on the basis of home-made movies made during the first year of life (Teitelbaum et al. 1998), (Teitelbaum et al. 2004). These clinical observations are consistent with a large body of evidence of subtle structural and functional abnormalities of cortical and subcortical neural systems involved in movement planning and execution, such as the prefrontal cortex, the basal ganglia and the cerebellum (see (Keller and Persico 2003), for a review).

\subsection{Ecological Approach}

The diagnosis of ASD is currently made at 3 years of age; Attention-Deficit Hyperactivity Disorder (ADHD) is always considered as an alternative diagnosis of "high functioning" autism; Tourette syndrome is diagnosed at age 7 or later. ASD is therefore a natural candidate for demonstrating the validity of novel approaches to early diagnosis. As shown in Fig. 1, infancy, i.e. the first 2-3 years of life before language development, represents an important temporal window for an early diagnosis of ASD.

The goal of our approach is twofold. On one hand, guided by neuroscientists, we develop technological platforms and methods to extract more information on perceptual and intersubjective capacities of human infants than is currently possible; this information could be later used for early diagnosis of developmental disorders. On the other hand, infancy provides us with an important window of opportunity to capture the mechanisms behind sensorimotor integration as these are just developing. Moreover, neurodevelopmental disorders are an important benchmark to highlight failures within such mechanism. Such a knowledge can be useful to neuroscientists to better understand the human brain functions involved in the 


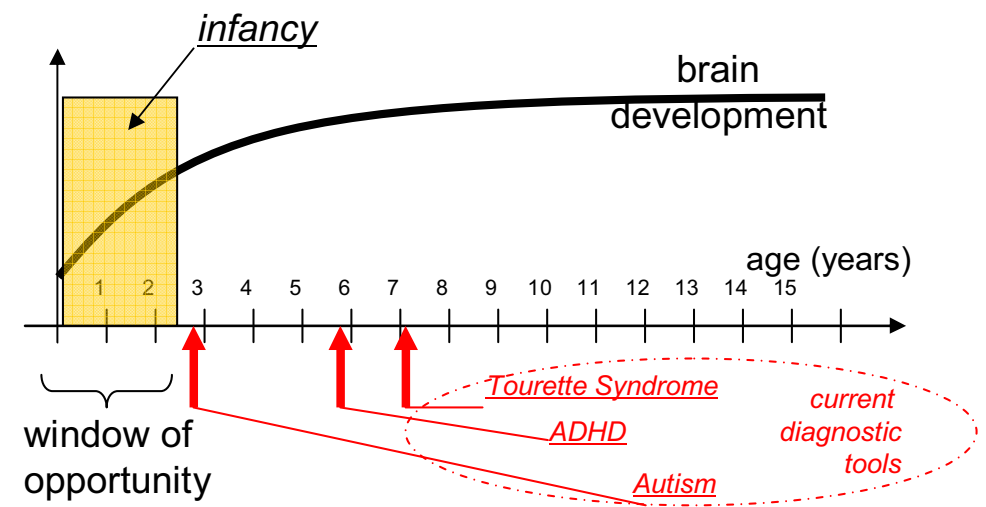

Fig. 1. Current diagnostic tools

sensorimotor integration but also to engineers, providing unique insights on how to build complex and adaptable artificial systems (Metta et al. 1999).

\section{Technology for Assessing Movement and Gaze}

\subsection{Motion Tracking}

Motion tracking can count on a host of different technological solutions, operating on entirely different physical principles, with different performance characteristics and designed for different purposes. As shown in (Welch and Foxlin 2002), there is not a single technology that can fit all needs. Each application defines the best technology to be implemented. In order to perform a selection, the main characteristics of available technologies are briefly summarized hereafter (see (Welch and Foxlin 2002) for details).

\section{Mechanical sensing:}

typically used for body motion capture; it uses angle and range measurements with the help of gears and bend sensors; very accurate but bulky, often limiting mobility.

\section{Optical sensing:}

several principles are available, typical systems are camera- based ones; position of markers in $3 \mathrm{D}$ space can be estimated very accurately within working volume (typically a few cube meters, depending on the number of deployed cameras); line-of- sight issues (i.e. the fact that body parts or other objects may occlude the visual scene of a camera, losing thus the sight of one or more markers) is a limiting factor; very expensive; often requires highly structured environments, at least when high accuracy is needed.

\section{Acoustic sensing:}

typically based on time-of-flight of ultrasound pulses between emitters and receivers; sound speed in air (about $340 \mathrm{~m} / \mathrm{s}$, resulting in sampling periods in the order of a few tens of milliseconds) is slow but still acceptable for sensing human (in particular infants) movements; line-of-sight issues are not as severe as for the optical technology; requires much less structured environments than optical trackers; suitable to be used in ecological conditions (e.g. kindergartens). 
(Geo)Magnetic sensing:

a first method is based on electromagnetic coupling between a source and several trackers; main drawbacks are that signal decays as $1 / d^{3}$ (where $d$ is the source-tracker distance) and is affected by the geomagnetic field; these devices are quite expensive and require a certain amount of structuring of the environment. A second method is electronic compassing; estimates heading and solely relies on the geomagnetic field, i.e. it does not require any artificial source and is therefore sourceless; measurements can be altered by ferromagnetic influence of surrounding objects.

\section{Inertial sensing:}

highly miniaturized accelerometers and gyroscopes are used to sense, respectively, acceleration (comprising the gravitational field) and angular velocity; used as inclinometers, accelerometers can sense the gravity vector, i.e. the 'vertical' direction, in this sense they are also sourceless.

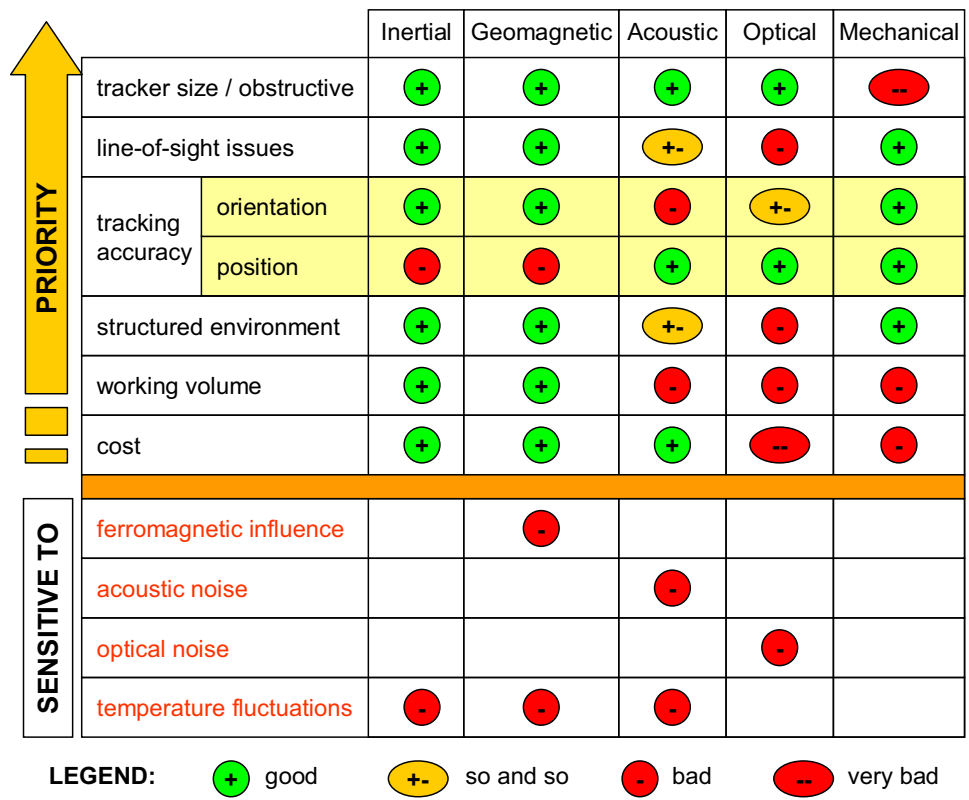

Fig. 2. Selection chart of different motion tracking technologies

In Fig. 2, a selection chart for the different available technologies is provided. For each available technology (columns) its suitability with respect to the performance characteristics of interest (rows) is indicated. Since our main purpose is developing technological tools that are either wearable by infants or embeddable into toys, the highest priority is given to technologies which are unobtrusive. This directly leads us to discard solutions involving mechanical trackers.

The second element considered for selection are the line-of-sight issues, since we are going to deal with infants, it is extremely difficult to perform experiments with technologies that are limited by the line of sight, a peculiarity of the optical technology which is only suitable to 
experiments with collaborative subjects who are somehow willing to 'act' in front of a camera. Line-of-sight issues are much less severe for the acoustic technology which is thus still appealing for movements analysis in infants.

The third element of the selection criterion is performance with respect to tracking accuracy. Here a distinction is made between tracking positions and tracking orientations. Measurement principles such as the time-of-flight (typically deployed in acoustic measurements) or camera-based tracking are inherently suitable to measure the distance of points (markers) and the origin of the measurement system (e.g. the source of acoustic waves or a camera etc...). Orientations can be inferred indirectly by estimating distances between two or more markers and the source of measurement. The larger the distance between two markers, the better the estimation of orientation. As dimensions shrink, as in the case of infants, accuracy of indirect orientation measurements also decreases (e.g. accurate tracking of the orientation of an infant's wrist can be problematic even without considering line-of-sight issues). Other technologies allow a direct measurement of orientations (for example inertial sensors used as inclinometers can sense deviations from the vertical axis while magnetic sensors used as compasses can sense deviations from the horizontal geomagnetic north direction) without requiring the positioning of multiple markers.

As long as orientation is concerned, inertial and magnetic technologies appear to be very appealing since: are highly unobtrusive due the availability of miniaturized off-the-shelf devices; do not suffer from line-of-sight issues; can provide high accuracy in orientation tracking are sourceless: do not require any structuring of the environment; have virtually unlimited working volume; are low-cost.

The bottom half of Fig. 2 shows, for each technology, the main limiting factors to a correct operation. Besides temperature, which affects any electrical device and that can be compensated in most of the cases, the real limiting factor for the magnetic technology is the presence of ferromagnetic materials. Common ferromagnetic objects such as iron parts of doors, chairs, tables etc... can produce local distortions of the geomagnetic field, causing thus errors in the estimations of orientations. As discussed in (Kemp et al. 1998), some care should be taken, when conducting experiments, to avoid large ferromagnetic objects in the surroundings. We found that this can be easily done in environments such as day-cares where, for safety reasons, all metals are usually avoided and typical materials used with children are wood, rubber and plastic.

\subsection{Gaze Tracking}

Devices for measuring eye movements are commonly referred to as 'eye trackers'. In general there are two types of techniques for monitoring eye movement (Young and Sheena 1975):

- 'eye-in-head' measurement: the sensing device is fixed on the head and therefore the eye position is measured in craniotopic coordinates;

- 'point of regard' or gaze: the sensors are located in the external environment and the eye position is measured in spatial coordinates.

These two kind of measurements are coincident when the head is kept in a fixed position. When the head is free to move, measurement of the head orientation is also required to derive gaze from craniotopic coordinates. Eye tracking methodologies can be classified in four categories:

1. Magnetic Induction Method (Search Coil)

2. Electro-Oculography (EOG) 
3. Photoelectric Methods: Infra-Red (IR) Oculography

4. Video-Oculography (VOG)

Each methodology is characterized by parameters such as range of measurement, sensitivity, linearity, accuracy, discomfort for the subject, interference with the field of view of the subject, tolerance to head movement.

\section{Magnetic Induction Method (Search Coil):}

The search coil technique has become the accepted standard for the measurement of 3D eye movement. This technique is based on the fact that a magnetic field induces a voltage in a coil (search coil) which is attached to the eye. The induced voltage has amplitude proportional to the sine of the angle between the coil axis and the magnetic field direction. The magnetic field is provide by coils mounted at the sides of a cubic frame. The dimensions of the sides of the frame can vary from few tens of centimeters to few meters, allowing to measure also other movements (i.e. eye-hand coordination). Robinson (Robinson 1963) was the first to apply this technique, using a coil secured to the eye by suction. Nowadays the search coil is embedded in a scleral contact lens. The lens is subject to slippage if the lens covers only the cornea. Eye movement is measured in absolute spatial coordinates. Head orientation can also be measured with a search coil mounted on the forehead, and orientation and movement of the eye within the head can be calculated from the orientation of the head and of the eye with respect to the magnetic field (Haslwanter 1995). Currently a number of different search coil systems are commercially available (e.g. by Skalar Instruments, C-N-C Engineering, Remmel Labs, etc.). Although the scleral search coil is the most precise eye movement measurement method (very high temporal and spatial resolution can be obtained with accuracy to about 5-10 arc-seconds over a limited range of about $5 \mathrm{deg}$ ), it is also the most intrusive method. Insertion of the lens requires care and practice and wearing the lens causes discomfort and risk of corneal abrasion or lead breakage. The requirements to stay in the center of the magnetic field precludes the use of search coils during many natural activities. Thus, this technique is mostly used for research purposes, it is not suitable for clinical routine.

\section{Electro-Oculography:}

First applications of electro-oculography are dated back to the '30s and are currently widely used both for clinical and research purposes. It relies on measurement of electrical potential differences between the cornea and the retina, discovered by DuBois-Reymond in 1849. Skin electrodes are positioned around the eye. The measured potential difference is proportional to the sine of the rotation angle of the eye. For small rotation the proportionality is almost linear; it decreases for higher angles of rotation (Byford 1963). The recorded potentials are in the range $15-200 \mu \mathrm{V}$, with nominal sensitivities of order of $20 \mu \mathrm{V} /$ deg of eye movement. The eye movement is measured in craniotopic coordinates and head movement during recording does not affect the measurement. The discomfort for the subject is limited and the measurement range is wide both for horizontal movements ( $\pm 70 \mathrm{deg}$ ) and for vertical movements ( $\pm 30 \mathrm{deg})$, even if the sensitivity decreases for lateral position of the eye. The most important advantage of this methodology is the possibility of recording eye movement with closed eyes, which is relevant requirement during some experimental protocol (e.g.. during sleeping phases). The main drawback of this technique are related to the nature of the potential recorded and to the artifacts due to the electrodes properties. As concern the potential, the resting corneoretinic potential (usually of the order of $0.4-1 \mathrm{mV}$ ) can be affected by lighting conditions of the environment and by the psycho-physical condition of the subject. The artifacts at the 
level of the skin electrodes relies on the contact resistance electrode-skin, on the oxidation and polarization of the electrodes.

\section{Infra-Red Oculography:}

Infra-red (IR) oculography is based on the recording of the light reflected by the eye when it is lighted with IR light beam. Since IR light is not visible, it does not interfere with the subject vision, moreover the IR detectors are not influenced by environmental lighting conditions. There are three categories of Infra-red (IR) oculography which use respectively: the corneal reflection, the Purkinje images and the track of the limb. Due to the construction of the eye, when a beam of IR light points to it, four reflections are formed on the eye, called Purkinje images (Cornsweet and Crane 1973): the first on the front surface of the cornea and it is called corneal reflection, the second image on rear surface of the cornea, the third on the front surface of the lens and the fourth on the rear surface of the lens. By detecting the corneal reflection and the pupil center and by using an appropriate calibration procedure, it is possible to measure the Point of Regard (gaze) on a planar surface on which calibration points are positioned. Two points of reference on the eye are needed to separate eye movements from head movements. The positional difference between the pupil center and corneal reflection changes with pure eye rotation, but remains relatively constant with minor head movements. The corneal reflection moves in the opposite direction of the eye respect to the pupil center. In other cases both the first and the fourth Purkinje images (Dual-Purkinje images eye trackers are detected. Both reflections move together through exactly the same distance upon eye translation but they move through different distances upon eye rotation. The third method based on photoelectric principle relies on the track of the limb (scleral-iris edge) of the eye by measuring the amount of scattered light. Most photoelectric systems must be mounted close to the eyes (i.e. EL-MAR tracking device), so they may restrict the field of view, moreover fast movements of the head can cause slippage of the device on the head leading to mis-alignment of the eye respect to the IR emitter and detector. There exist also external device and a support for keeping the head fixed is needed (i.e. Tobii eye tracker). The range of measurement of the photoelectric eye tracker is not higher then \pm 30 deg in the horizontal plane and \pm 20 deg in the vertical plane.

\section{Video-Oculography:}

Video systems for measuring ocular movements are based on the analysis of images recorded by cameras. This technique, introduced in the ' 80 s, quickly improved in terms of performances and reliability thanks to the technological development of digital cameras and computer powerful. The Video Oculography (VOG) provides directly a digital output. Several algorithms are available for the pupil detection in an image frame and pupil centroid coordinates extraction, nevertheless environmental lighting conditions can affect the automatic detection (Eizenman et al. 1984) (Landau 1987). Thus, IR light is used together with video recording, so that the pupil appears brighter. This technique is called Pupil Center/Corneal Reflection (PC/CR) because the IR light produces also the Purkinje images, mentioned before. As in IR Oculography, also VOG can be realized both as wearable device (DiScenna 1995) and provides measurements in craniotopic coordinates or external device and provides measurements in spatial coordinates. Head-mounted system (i.e. EyeLink) can be worn without too much discomfort. High resolution and high frame rate CCD and CMOS cameras are used. Reduced dimensions and weight of the actual cameras allow to position them in such a way that they interfere as less as possible with the field of view of the subject (Babcock and Pelz 
2004), (Pelz et al. 2000). The measurement range for VOG systems can exceed \pm 30 deg in horizontal plane and \pm 20 deg in the vertical plane; eye tracking can be executed both on-line and off-line. The drawback is that these systems have a low acquisition rate, in general 50-60 $\mathrm{Hz}$, not suitable for recording fast eye movement such as saccadic movements, but suffices for smooth pursuit eye movements. External camera systems can go up to $1000-1250 \mathrm{~Hz}$ and have an accuracy of $0.01 \mathrm{deg}$. External camera are generally positioned under the screen of a computer, used for calibration and for specific visual stimuli. Head movements are tolerated if the eye is kept in the field of view of the camera. There are devices which include systems of pursuit of the subject and the camera orients automatically so that the eye of the subject is always in its field of view.

In Table 1 the relevant parameters of the eye tracking techniques presented are summarized.

\section{Instrumented Toys and Wearable Devices}

Virtually any toy, tool or piece of garment used by children could be a good candidate to host all sorts of technology and 'see what comes out' when the child wears it or plays with it.

Our approach is based on a closed-loop dialogue between neuroscientists and bioengineers. In the following, two platforms are presented which specifically address two domains of interest in child's development: spatial cognition and social behavior.

For both platforms, functional specifications are derived from protocols of experiments of interest for neuroscientists. The aim is twofold. On one side we wish to provide neuroscientists with novel technological platforms for the unobtrusive and ecological assessment of behavioral development in infants. On the other side, these platforms should enable/facilitate the transition from research to clinical practice.

\subsection{Assessing Spatial Cognition Skills}

By the end of the first year of life, infants start to pile-up blocks, put lids on cans and insert objects into apertures. Through these activities, the child learns to plan actions that involve more than one item. The ability to solve such problems reflects the child's spatial, perceptual and motor development. In particular, the representational ability to imagine objects in different positions and orientations must be in place before various objects can be fit into apertures.

Recent studies by Ornkloo and von Hofsten (Ornkloo and von Hofsten 2007) show developmental curves, based on statistical rates of success of object-fitting tasks, relative to children aged 14-26 months old.

Specifically, the tasks consisted of inserting cylinders with different cross-sections into a box with similar holes on its lid, see Fig. 3 (top). All the objects had similar dimensions, $1 \mathrm{~mm}$ smaller than the apertures. Different cross-sections were used whose circumference was approximately the same but varied with respect to the number of possibilities they fit into a corresponding aperture, as also reported in Fig. 3 (bottom).

Based on visual inspection of video recordings, the data analysis consisted (among other things) in assessing horizontal and vertical pre-adjustments. In particular, the outcome was yes/no (i.e. successful or unsuccessful) based on the alignment errors between the object and the box. Both the vertical error (angular misalignment between the longitudinal axis of the object and verticality) and the horizontal error (angular misalignment between the orientations of the cross- section and the aperture) were estimated (from the videos). Results showed that successful solution was associated with appropriate preadjustments before the hand arrived with the block to the aperture; in particular it has been proved that the preadjustments can be considered appropriate for misalignments lower than $30 \mathrm{deg}$. 


\begin{tabular}{|c|c|c|c|c|}
\hline & Search coil & $\begin{array}{l}\text { Electro- } \\
\text { Oculography }\end{array}$ & $\begin{array}{l}\text { Infra-Red } \\
\text { Oculography }\end{array}$ & $\begin{array}{l}\text { Video } \\
\text { Oculography }\end{array}$ \\
\hline $\begin{array}{l}\text { Measurement } \\
\text { Typology }\end{array}$ & $\begin{array}{l}\text { Absolute spatial } \\
\text { coordinate }\end{array}$ & $\begin{array}{l}\text { Craniotopic } \\
\text { coordinates }\end{array}$ & $\begin{array}{l}\text { Head-mounted: } \\
\text { craniotopic } \\
\text { coordinates; } \\
\text { External device: } \\
\text { spatial } \\
\text { coordinates }\end{array}$ & $\begin{array}{l}\text { Head-mounted: } \\
\text { craniotopic } \\
\text { coordinates; } \\
\text { External device: } \\
\text { spatial } \\
\text { coordinates }\end{array}$ \\
\hline $\begin{array}{l}\text { Range of } \\
\text { measurement }\end{array}$ & $\begin{array}{l} \pm 90 \text { deg for all } \\
\text { 3D space }\end{array}$ & $\begin{array}{l} \pm 70 \text { deg in } \\
\text { horizontal plane } \\
\pm 30 \text { deg in } \\
\text { vertical plane }\end{array}$ & $\begin{array}{l} \pm 30 \text { deg in } \\
\text { horizontal plane } \\
\pm 20 \text { deg in } \\
\text { vertical plane }\end{array}$ & $\begin{array}{l} \pm 30 \text { deg in } \\
\text { horizontal plane } \\
\pm 20 \text { deg in } \\
\text { vertical plane }\end{array}$ \\
\hline $\begin{array}{l}\text { Temporal } \\
\text { Resolution }\end{array}$ & $\begin{array}{l}\text { linked to A/D } \\
\text { conversion, } \\
500-1000 \mathrm{~Hz} \\
\text { (depends on } \\
\text { software and } \\
\text { hardware } \\
\text { instrumentation) }\end{array}$ & $\begin{array}{l}\text { linked to A/D } \\
\text { conversion, } \\
500-1000 \mathrm{~Hz} \\
\text { (depends on } \\
\text { software and } \\
\text { hardware } \\
\text { instrumentation) }\end{array}$ & $\begin{array}{l}\text { linked to A/D } \\
\text { conversion, } \\
500-1000 \mathrm{~Hz} \\
\text { (depends on } \\
\text { software and } \\
\text { hardware } \\
\text { instrumentation) }\end{array}$ & $\begin{array}{l}\text { Depends on } \\
\text { camera frame } \\
\text { rate: from } 30 \mathrm{~Hz} \\
\text { to } 1000-1250 \mathrm{~Hz}\end{array}$ \\
\hline $\begin{array}{l}\text { Spatial } \\
\text { resolution }\end{array}$ & $0.01 \mathrm{deg}$ & $1-1.5 \mathrm{deg}$ & $0.1 \mathrm{deg}$ & $0.1 \mathrm{deg}$ \\
\hline Discomfort & High & Limited & Limited & Limited \\
\hline $\begin{array}{l}\text { Interference } \\
\text { with the } \\
\text { subject field } \\
\text { of view }\end{array}$ & None & None & $\begin{array}{l}\text { Head-mounted } \\
\text { device can } \\
\text { interfere with the } \\
\text { field of view }\end{array}$ & $\begin{array}{l}\text { Head-mounted } \\
\text { device can } \\
\text { interfere with the } \\
\text { field of view }\end{array}$ \\
\hline $\begin{array}{l}\text { Tolerance to } \\
\text { head } \\
\text { movement }\end{array}$ & $\begin{array}{l}\text { - Head has to be } \\
\text { in the center of } \\
\text { the magnetic } \\
\text { field } \\
\text { - Additional } \\
\text { search coil on the } \\
\text { forehead }\end{array}$ & $\begin{array}{l}\text { Not affected by } \\
\text { head movement }\end{array}$ & $\begin{array}{l}\text { External device: } \\
\text { low tolerance to } \\
\text { head movements }\end{array}$ & $\begin{array}{l}\text { - Head } \\
\text { movements are } \\
\text { tolerated when } \\
\text { eye is kept in the } \\
\text { field of view of } \\
\text { the camera; } \\
\text { - Additional } \\
\text { sensors allow to } \\
\text { re-orientate the } \\
\text { camera }\end{array}$ \\
\hline Other Notes & $\begin{array}{l}\text { - limited } \\
\text { recording time } \\
\text { and risk of } \\
\text { corneal abrasion } \\
\text { or lead breakage } \\
\text { - lens slippage }\end{array}$ & $\begin{array}{l}\text { - Measurement } \\
\text { with closed eyes } \\
\text { (during sleeping) } \\
\\
\text { - Skin electrodes } \\
\text { artifacts } \\
\text { - Resting } \\
\text { corneo-retinal } \\
\text { potential } \\
\text { variability }\end{array}$ & $\begin{array}{l}\text { - Not suitable } \\
\text { when the subject } \\
\text { wears glasses or } \\
\text { contact lens }\end{array}$ & \\
\hline
\end{tabular}

Table 1. Comparison of different gaze tracking technologies

\subsubsection{Block-Box Platform}

Inspired by such experiments and based on our previous experience with sensorized toys (Campolo et al. 2007), we developed a sensorized core, shown in Fig. 4 (top), for the cylindrical 


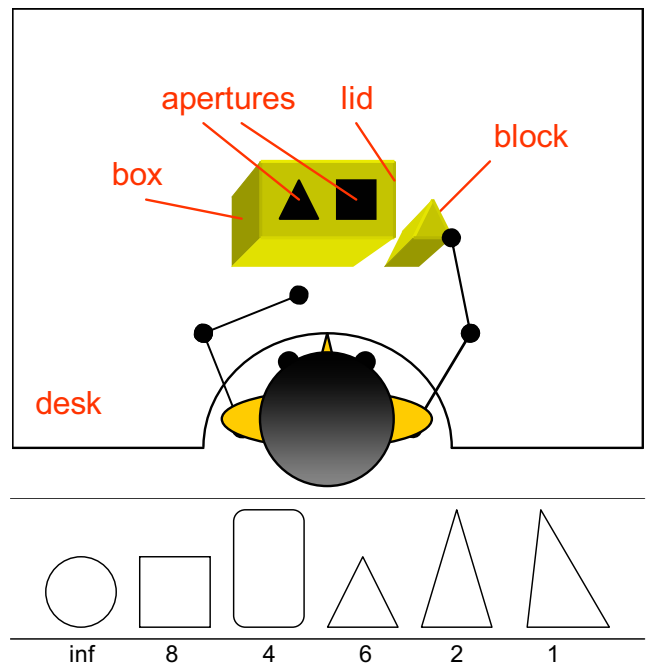

Fig. 3. Block-box experimental scenario (top). Different cross- sections (bottom) for the cylindrical blocks and the relative number of insertion possibilities ('inf' means infinite), readapted from (Ornkloo and von Hofsten 2007)

objects with various cross-sections, shown in Fig. 4 (bottom).

In particular, we found that from an ecological perspective, the sourceless estimation of objects orientation via inertial and magnetic sensors is especially suited to this application. Accelerometers can in fact be used to measure tilt while magnetometers can be used as compass to measure horizontal misalignments. Gyroscopes are required to compensate for non-static effects. Further details on the filter used to estimate orientation from the sensors raw data is described in (Campolo et al. 2008).
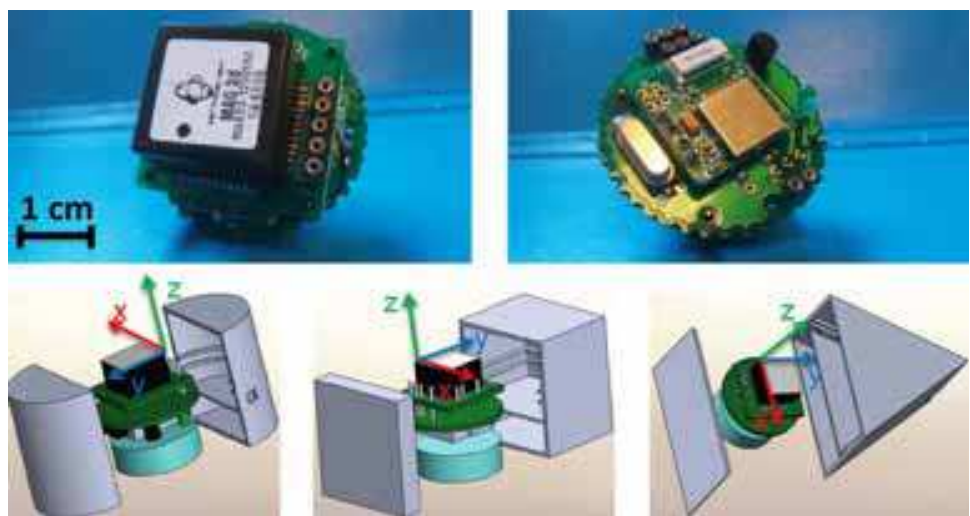

Fig. 4. Kinematics sensing unit (top left). Bluetooth transmitting unit (top right). Examples of assemblies of electronics and batteries for shells with different cross-section (bottom). 
By considering the requirements of the experimental setup and protocol of the above mentioned study (Ornkloo and von Hofsten 2007), the functional specifications of the block-box platform can be resumed as follows:

- the device (electronic core and power supply) should be embeddable in solids with different shapes with a "grasping size" less than $5 \mathrm{~cm}$;

- the sensor unit should consist of sourceless sensors for orientation tracking;

- the transmission unit should assure a bi-directional wireless communication to a remote workstation few meters far from the experimental scenario;

- the batteries should provide power supply for at least 2 hours of continuous use;

- the overall weight of the toy should not exceed the 50-60 grams.

According with these specifications, the block-box sensorized toys have been designed to be as compact and light as possible. In particular, the platform mainly consists of a compact $(17.8 \mathrm{~mm} \times 17.8 \mathrm{~mm} \times 10.2 \mathrm{~mm})$, micro-fabricated 9-axis inertial-magnetic sensor (model MAG02-1200S050 from Memsense Inc.). The device is designed to sense $\pm 2 \mathrm{~g}$ accelerations, $\pm 1200 \mathrm{deg} / \mathrm{sec}$ angular rates, \pm 1 Gauss magnetic fields, all within a $50 \mathrm{~Hz}$ bandwidth. The sensors are coupled with a multi-channel, 12 bits AD converter (model MAX1238 from Maxim Inc.) which can retransmit converted data over a 4-wires I2C bus. For our application, we sample each of the 9 channels at 100 Samples/sec rate. Such data are collected and rearranged in a specific message format by a microcontroller (PIC16F876A from Microchip Technology Inc.) and then retransmitted via a bluetooth module (Parani-ESD200 from Sena Technologies Inc.). Finally, two 3.6V Li-Ion Rechargeable batteries (LIR3048 from Powerstream Inc.) are used in series, in order to guarantee approximately two hours of autonomous operation. Data transmitted over the bluetooth interface are collected by a nearby PC, for later data analysis. Fig. 5 represents the overall architecture of the block-box platform. As it can be noted, we decided to arrange the different components into two separate electronic boards (a sensor board and a transmission board), which are connected through the I2C bus. This solution makes the system modular, allowing us to easily change the sensor unit or put together several sensors that share the same bus.

In Fig. 6 the electronic CAD designs (left) and the real pictures (right) of the sensor (top) and the transmission (bottom) boards are shown. Fig. 7 reports the overall aspect of the electronic core of the Block-Box platform with the actual dimensions.

\subsubsection{In-Field Calibration of Inertial-Magnetic Sensors}

Magnetometers are meant to sense the geomagnetic field and provide its components $\left[b_{x}, b_{y}, b_{z}\right]^{T}$ along the $\hat{x}, \hat{y}$ and $\hat{z}$ axes of the sensing device itself (such axes move with the moving frame). Similarly, the accelerometers are meant, in static conditions, to read out the components of the gravitational field $\left[g_{x}, g_{y}, g_{z}\right]^{T}$ along the same axes.

Calibration of such sensors is straightforward when one can reliably count on precision alignment procedures, e.g. in a laboratory setting. In (Campolo et al. 2006), a procedure for infield calibration of magnetometric sensors was presented which does not rely on previous knowledge of magnitude and direction of the geomagnetic field and which does not require accurately predefined orientation sequences. Such a method can be applied to accelerometers as well and is especially suited for clinical applications. The procedure relies on the fact the geomagnetic (or gravitational) field has constant components in the fixed frame. As the orientation of the sensors vary, the components in the moving frame also vary but the magnitude 


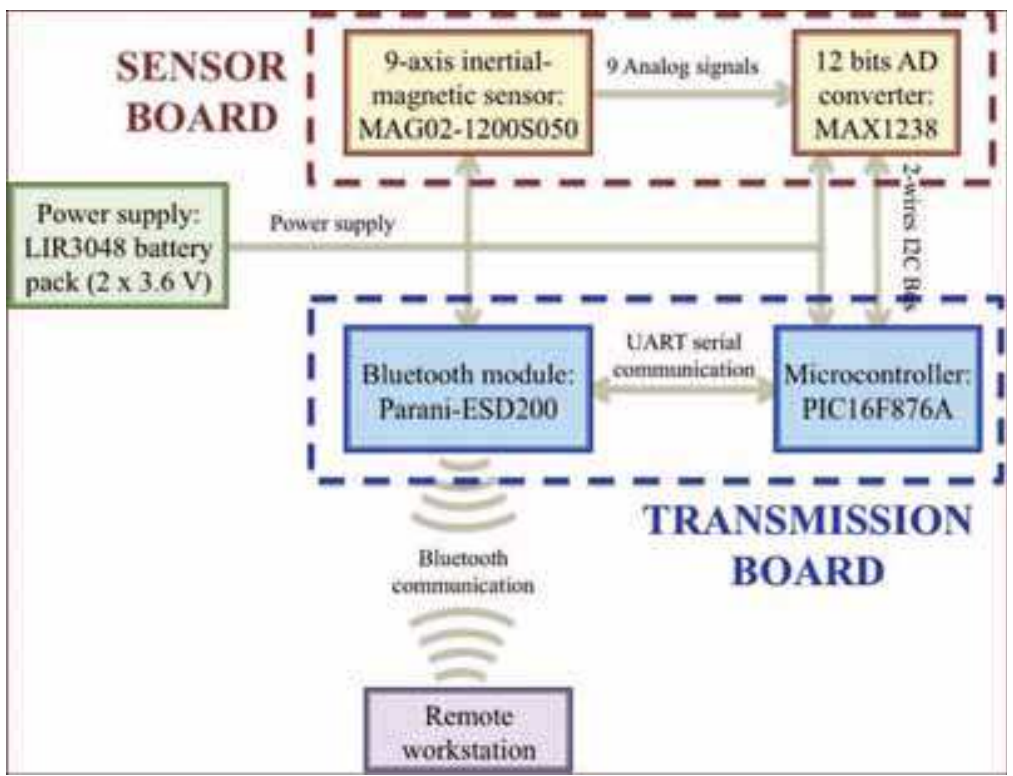

Fig. 5. Block-Box Platform architecture.

of the field keeps constant, i.e. the components are bound to lie on a sphere. Readouts from non-calibrated sensors are therefore bound to lie on an ellipsoid, see (Campolo et al. 2006) for details. Via the least-square method it is possible to robustly estimate the centroid and semiaxes length of the ellipsoid which coincide with the calibration parameters (gain and offsets for each axis).

Based on this method, a calibration protocol was devised to provide a sufficient number of measurements for the algorithm to robustly converge. The instrumented toy (of whatever shape) is secured inside a wooden box, shaped as a parallelepiped, so that the toy does not move as the box is displaced around.

\section{Magnetometers:}

as in Fig. 8-a, the box is placed on a table and an approximately 360 deg rotation (no need to be accurate) is performed by keeping one face of the box always parallel and in contact with the table. The same procedure is repeated for four different faces.

\section{Accelerometers:}

as in Fig. 8-b, the box is placed on a table and smoothly (i.e. avoiding shocks) tilted by 90 deg along one edge, this is repeated four times ${ }^{1}$ until the box returns in the initial position. The whole procedure is repeated with a different initial position.

\section{Gyroscopes:}

the procedure is similar to the one deployed for the accelerometers.

\footnotetext{
${ }^{1}$ Each time on a different edge: once a $90 \mathrm{deg}$ rotation is performed along one edge, the next edge is the non-consecutive one which also makes contact with the table.
} 

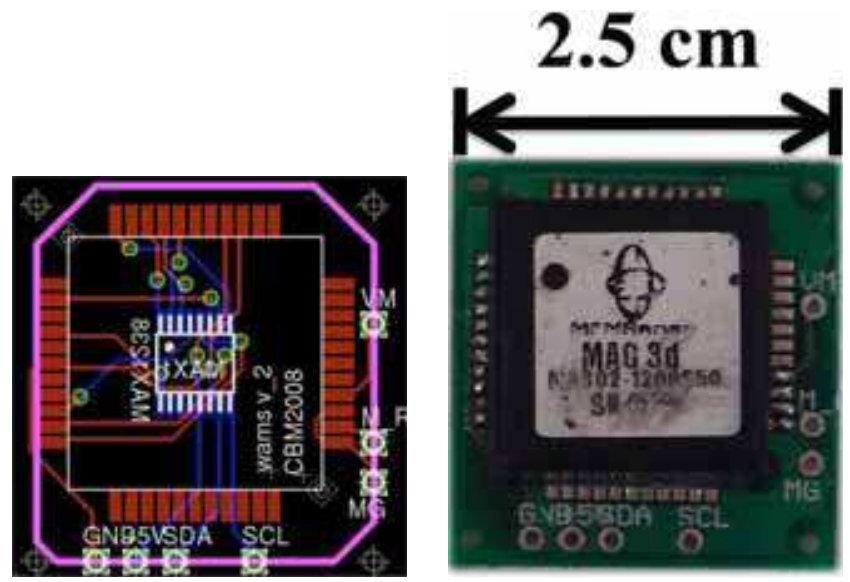

\section{$3.5 \mathrm{~cm}$}
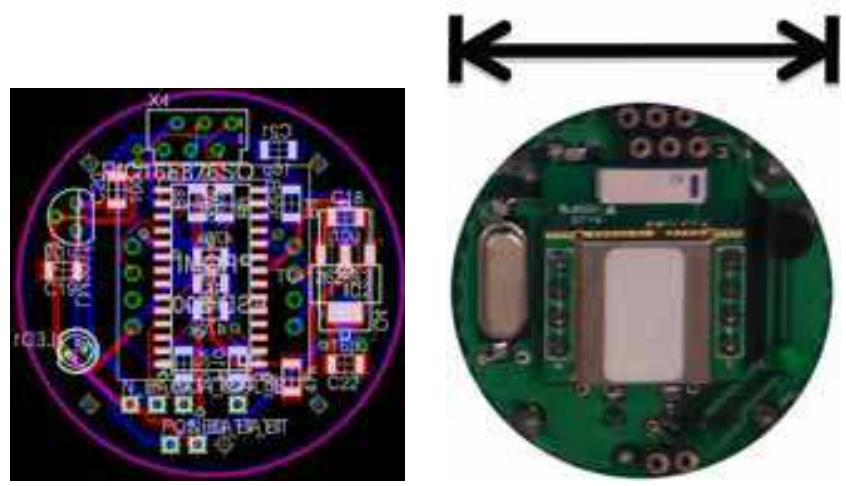

Fig. 6. The electronic CAD designs (left) and the real pictures (right) of the sensor (top) and the transmission (bottom) boards.

Measurements derived from a calibration sequence are shown in Fig. 8-c and Fig. 8-d, respectively for the magnetometers and for the accelerometers. The least-squares algorithm is then used to derive the best fitting ellipsoids (one for the magnetometers and one for the accelerometers) whose surfaces contain the two sets of measurements.

As previously mentioned, since the geomagnetic field is constant, its components in the moving frame are bound to lie on the calibrating ellipsoid, not only during the calibration sequences but for every possible movement. For this reason, also movements performed during the regular use of the toy, i.e. when the infants plays with it, can be used for updating the calibration parameters, or at least for an on-line check. Similar procedures apply to accelerometers, paying attention to consider only the quasi-static movements, i.e. when accelerations of the movement itself are negligible with respect to gravity. Details about 'in-use' calibration can be found in (Lotters et al. 1998). 

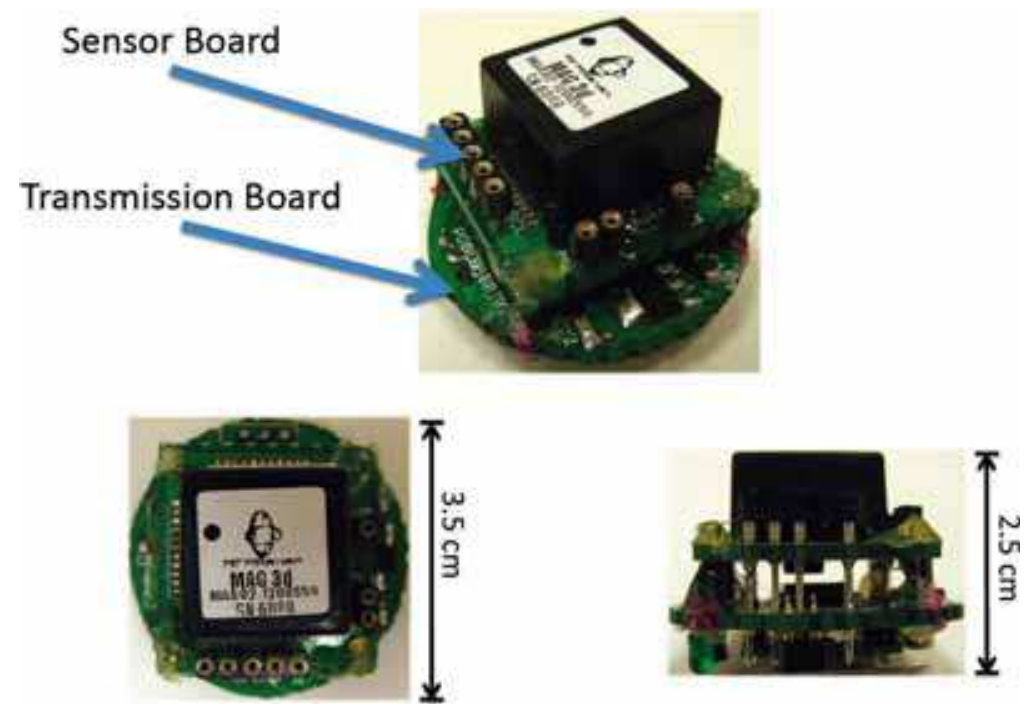

Fig. 7. Electronic core of the Block-Box platform with the actual dimensions.

\subsection{Assessing Spontaneous Movements}

Infants show a large variety of spontaneous movement patterns. Such movements are called spontaneous to tell them apart from reflex movements because they are endogenously generated by infantsŠ nervous system without any external sensory input.

While reflexive movements allow a detailed study of a stable, quantitative relationship between sensory input and reflexive motor output, spontaneous motility could be regarded as the expression of spontaneous neural activity, so it is an excellent marker of neural dysfunction caused by brain impairments during the first year of life (Prechtl and General 2001). Among several spontaneous motor patterns, movements that appear to be more effective for functional assessment of infantsS nervous systems are complex sequential movements of arms, legs, neck, and trunk called General Movements (GMs): they are characterized by rotations along the axis of the limbs and slight changes in the direction of movements (see (Einspieler et al. 2005) for a review).

If the nervous system is impaired, GMs change their quality or even disappear: they lose their complex character and involve a reduced number of limbs in monotonous sequences; they appear to be rigid and less smooth because generated by the almost simultaneous contraction of all limb and trunk muscles. Such alterations are considered to be highly predictive for later neurological impairments like Cerebral Palsy (CP).

During the assessment phase, infants with bare arms and legs are videotaped in supine position. The duration of the recording depends on the age of the infant: to collect at least three GMs, $1 \mathrm{~h}$ recording is necessary with pre-term infants while 10 minutes are enough from term age onward. The same infant is videotaped at different ages: from 2 to 3 recordings during the pre-term period; one recording at term or early post-term age or both, and at least one recording between 9 and 15 weeks post-term. After each recording session, a trained clinician reviews the videotape looking for GMs sequences that are copied onto an assessment tape. The observed GM and the week of development are reported on a table called individual 


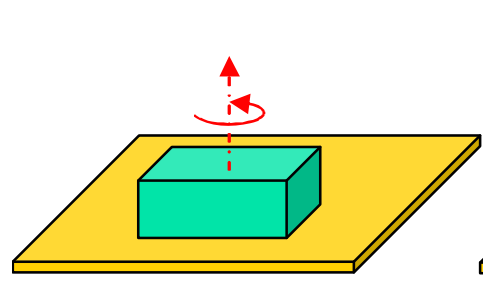

a)

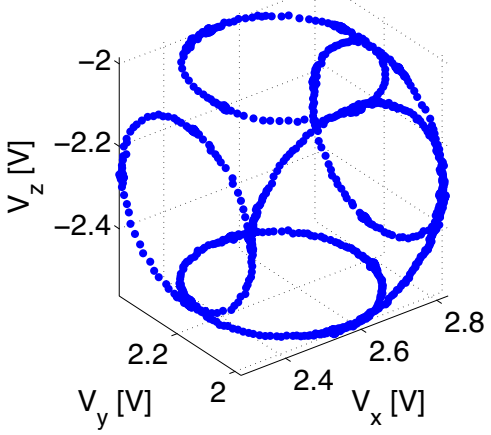

c)

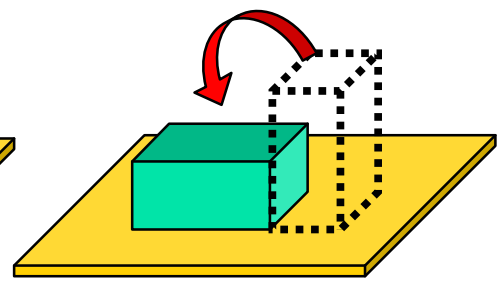

b)

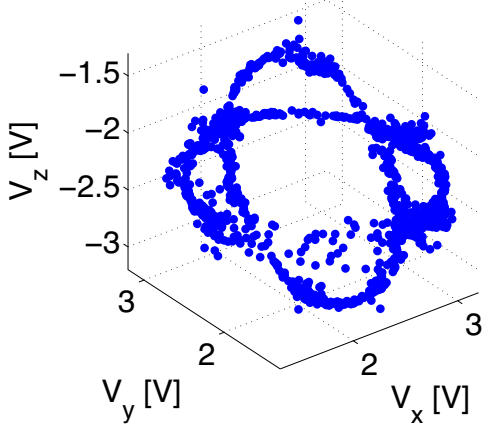

d)

Fig. 8. Calibration sequences for magnetometers (a) and accelerometers (b). Plots of the measurements (i.e. voltages $V_{x}, V_{y}$ and $V_{z}$ from the triaxial sensors) derived from the calibration sequences for the magnetometers (c) and the accelerometers (d).

developmental trajectory. Such table is used to assess the risk of developing lateral neurological impairments. 11 studies on 358 infants assessed by 90 observers revealed an inter-rater reliability between $89 \%$ and $93 \%$ (Einspieler et al. 2005).

\section{Wrist and Ankle Movement Sensor (WAMS)}

GMs assessment protocol collects qualitative information on motor behavior of infants during their first year of life. In order to match the individual developmental trajectory with some quantitative objective information we proposed a magneto-inertial wearable device for ecological behavioral analysis of infants' motor behavior called Wrist and Ankle Movement Sensor (WAMS) (Taffoni et al. 2008). Such a device, which allows gathering objective information such as the angle of rotation along the axis of the limb, its velocity, smoothness, acceleration etc., could improve the predictive validity of GMs, thus reducing the inter-observer disagreement.

Because the target is assessing infants' movement, it is important to reduce size and weight of the device as much as possible. Although there are several commercial solutions, they are often exceedingly large and bulky. According to anthropometric data, the maximal linear length of a wearable device should not exceed $2.5 \mathrm{~cm}$ and should not weight more than 20 grams.

In order to estimate a proper scale range of the sensor, preliminary quantitative experiments have been conducted. A three-cameras $(500 \mathrm{~Hz}$ ) motion capture system was used (Qualysis 
Motion Capture Systems Inc., SWE) to obtain position-time data from both arms of infants and evaluate frequency and dynamic content of their spontaneous movements. Three reflective markers were placed on the head and two on both hands. In Fig. 9-A data from one-week-old infant are shown.

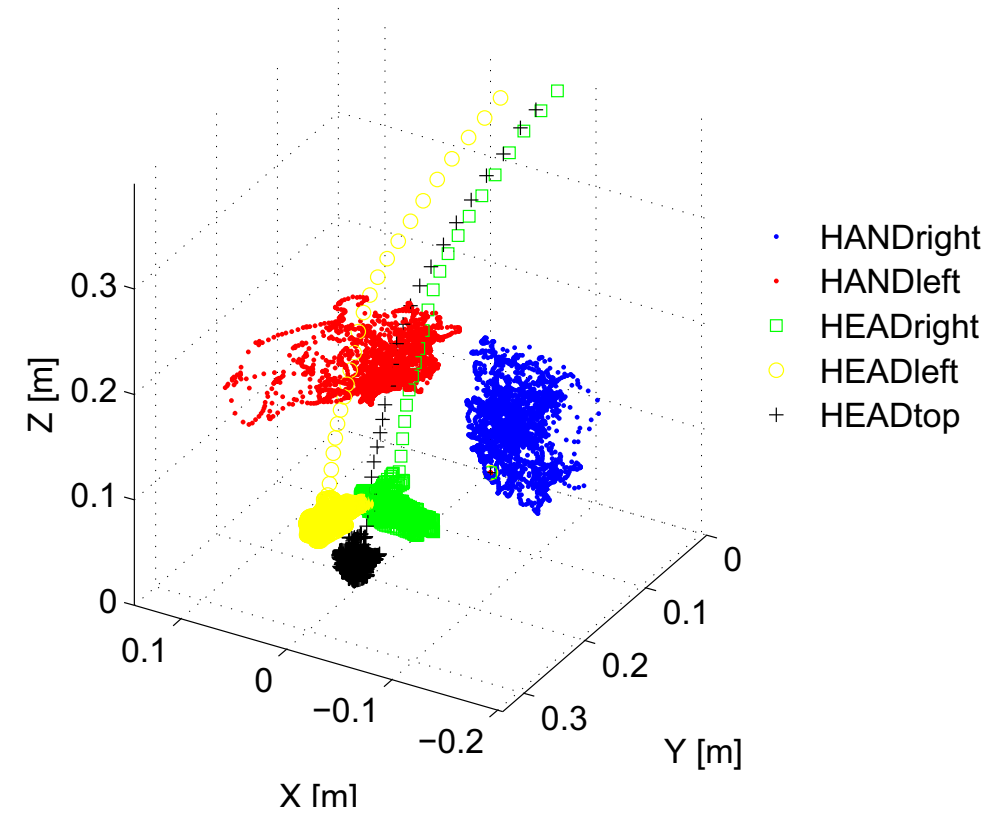

Fig. 9. Representative data relative to head-hands kinematics of a one week old infant

Probability Density Function (PDF) of several sets of experimental data was computed to estimate the dynamic range for the accelerometer sensors, showing that a $\pm 2 g$ range would be sufficient to capture infants' kinematics. As for the magnetometers, since only the geomagnetic field (about 0.6 gauss) needs to be measured, the full measurement range should be in the order of \pm 1 Gauss. Saturation of gyroscopes would result in a problematic loss of tracking and therefore, given several commercially available gyroscopes with full scale ranging from $\pm 150 \mathrm{deg} / \mathrm{s}$ to $\pm 1200 \mathrm{deg} / \mathrm{s}$, the maximum scale range ( $\pm 1200 \mathrm{deg} / \mathrm{s})$ was selected.

In line with such specification a sampling frequency of $250 \mathrm{~Hz}$ was selected. This frequency is between the sampling frequency of other magneto-inertial commercially available systems (typically $100 \mathrm{~Hz}$ ) and the frequency of the optical devices (up to $500 \mathrm{~Hz}$ ).

A preliminary analysis of commercial magneto-inertial sensors currently available highlighted a triaxial magnetometer, accelerometer and gyroscope analogue sensor from Memsense (MAG02-1200S050) that matches the technical requirements previously defined. Although there are several highly miniaturized components available off the shelf, we have chosen to use a microfabricated device integrating all the required sensing capability for two main reasons: 1) a more efficient packaging; 2) a more reliable axis orientation. Not orthogonal sensible axes directly translate in errors during orientation tracking. In order to simultaneously 
collect data from both arms and legs we propose a Body Area Network (BAN) of four sensors: two for arms and two for legs. Unlike common BAN (Jovanov et al. 2005) we chose to use a wired configuration in order to reduce the weight of each sensor: wired communication allows not to include the battery onboard, and therefore to reduce the weight. For such reasons each sensing unit is provided with a low power, 12-channels, 12 bits AD Converters with an I2C compatible two wires serial interface. In this way only 4 wires are required: 2 for data and 2 for supply, which is in a remote master station. In this station a microcontroller PIC16F876A collects data from each WAMS (identified by a unique address) and retransmits via RS232 to a PC where such data are stored. The electronic board is embedded into a soft silicon rubber structure used both for electrical passivation and for comfortable contact with the infant's skin. A Velcro strap is used to fix such sensors to the infant's limbs. Figure 10 shows the first WAMS prototype: total weight less then 14 grams and volume within $2.5 \times 2.5 \times 1.5 \mathrm{~cm}^{3}$.

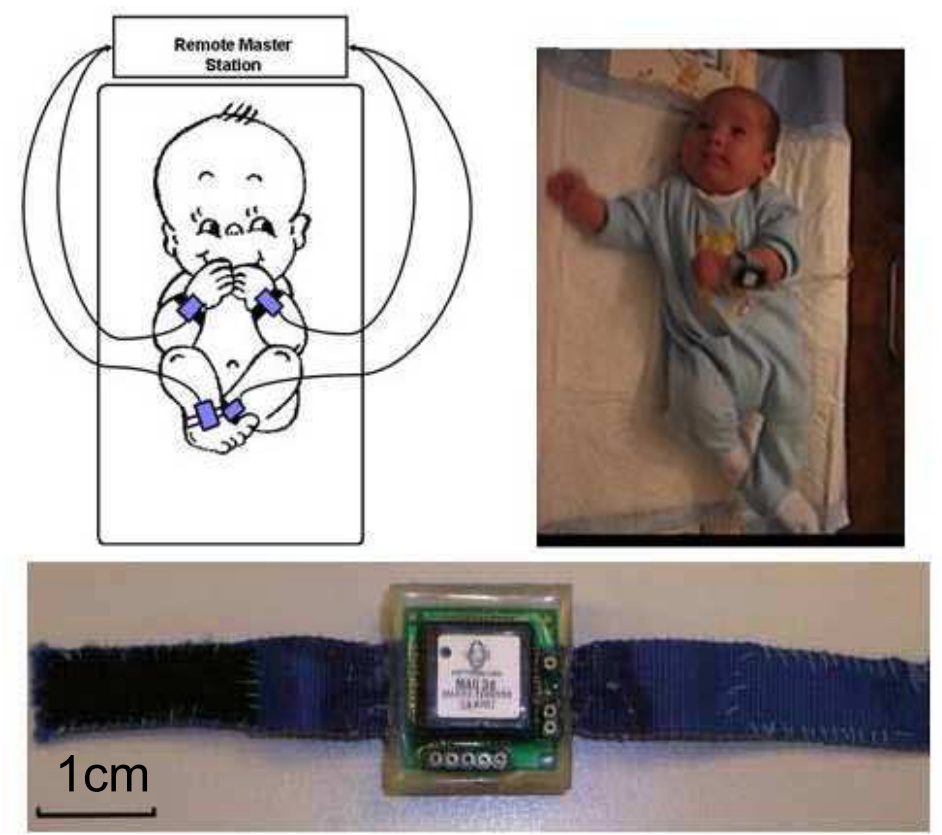

Fig. 10. Typical experimental scenario. (A). Example of use on a 3 months old baby (B). First WAMS prototype $(\mathrm{C})$.

\subsection{Assessing Social Attention}

Poor sensorimotor integration has long been addressed as a cause of motor and social problems in developmental disorders such as ASD (Trevarthen and Daniel 2005). Failure in orienting towards occurring social stimuli (e.g. facial expressions, speech, gesture) represents one of the earliest and most basic social impairments in autism and may contribute to the lateremerging social and communicative impairments (Dawson et al. 2004). Early social exchanges require rapid shifting of attention between different stimuli. Impairments in social orienting can alter the developmental pathway of young children by depriving them of appropriate social stimulation (Mundy and Neal 2001). 


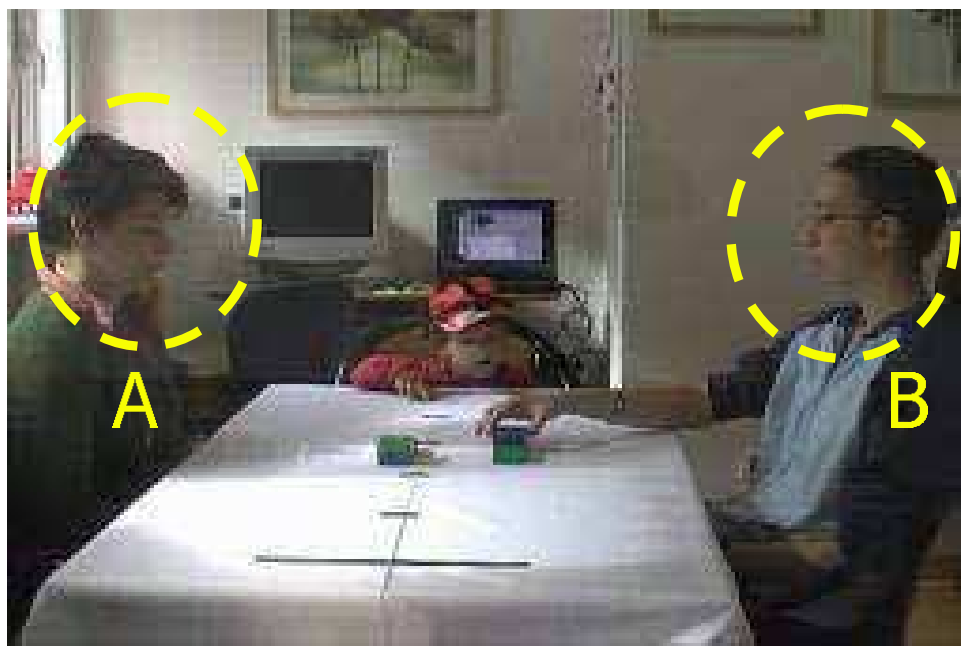

Fig. 11. Social situation.

A social protocol to study the response of a child to social stimuli was devised by G. Stenberg (Dept of Psychology, University of Uppsala, Sweden). As shown in Fig. 11 (top), a child sits at a table observing two adults sitting approximately $45 \mathrm{deg}$ left and right. The adult subjects engage in a conversation while alternatively positioning blocks in front of the child. Observing the video-recordings, the psychologist would then take note (frame by frame) of when the child looks at the adult subjects, when at the blocks, whether the child can anticipate the 'next move' of an adult subject (based on the clues provided in the conversation). Indeed a lengthy process, requiring hours to rate a few minutes of an experiment.

\subsubsection{Audio-Visuo-Vestibular Cap platform}

Early diagnosis is also based on the possibility of screening a large number of children and automatic, or at least semi-automatic, methods would be very valuable.

To this end, we developed the multimodal Audio-Visuo-Vestibular Cap (AVVC) platform, shown in Fig. 12, specifically devised to assess sensorimotor integration in social orienting behaviors in very young children, from 6 to 24 months of age. In particular it allows monitoring the child's gaze and facial expressions, monitoring the head kinematics, localize in sound stimuli with respect to the child.

\subsubsection{Gaze and facial expressions}

in the current version, a lightweight eye-cam $\left(1 / 4^{\prime \prime}\right.$ CMOS sensor, $640 \times 480$ resolution, 30 frames/sec) is mounted on the beak of the cap monitors. A mini-objective (model RE-025S, $2.5 \mathrm{~mm}$ focal length, $84 \mathrm{deg}$ diagonal field-of-view, $57 \mathrm{deg}$ vertically and 71 deg horizontally) is used to keep the face of the child on focus. This provides still images of the face, independently of the head movements, and in particular allows monitoring the gaze. 


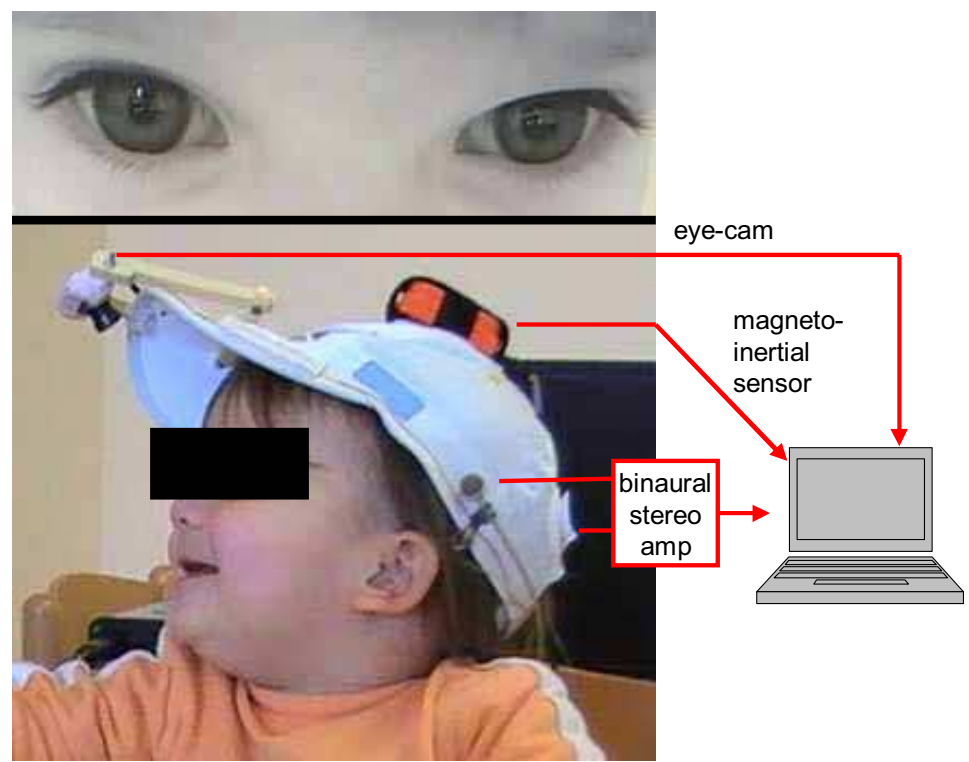

Fig. 12. Audio-Video-Vestibular-Cap (AVVC).

\subsubsection{Head kinematics}

is monitored via a commercial magnetic-inertial sensor (MTx-28A33G25 device from XSens Inc.; static orientation accuracy < 1deg; bandwidth $40 \mathrm{~Hz}$; sampling rate $100 \mathrm{~Hz}$ ). Similarly to the human vestibular system, the inertial sensors can be used to estimate tilt with respect to gravity as well as angular velocity of the head. Furthermore, the magnetic sensors, used as a compass, can determine the amount of head rotation on the horizontal plane.

\subsubsection{Direction of sound stimuli}

is an important clue in social behavior. Humans localize sound in space via interaural time differences (ITD) and intensity level differences (ILD), over various frequency ranges (Blauert 1997). To this end, we use a pair of microphones (MKE 2-ew Gold, Levalier, sampled in stereo audio quality at $44.2 \mathrm{kHz}$ ) mounted on the cap in correspondence of the ears of the child. The ITD of sound arrival at the two microphones can be estimated based on the generalized crosscorrelation algorithm (Rucci and Wray 1999).

\subsubsection{Ecological Calibration of the Binaural Microphones for the AVVC}

An ITD/angle relationship is required to determine the angular position of a sound source with respect to the head once the ITD is estimated. Typical calibration procedures involve relative positioning of a sound source with respect to a pair of binaural microphones. For various angular positions, the relative ITDs are estimated and the ITD/angle relationship is thus experimentally determined.

For the AVVC platform this is a challenging task as calibration can only be done after the child wears the cap, mainly for two reasons: $i$ ) the ITD also depends on how the sound propagates through the head of the child, therefore the cap must be worn; ii) the cap is manually fit 
onto the child's head and this procedure is neither accurate or repeatable, i.e. a calibration is required every time the child wears the cap.

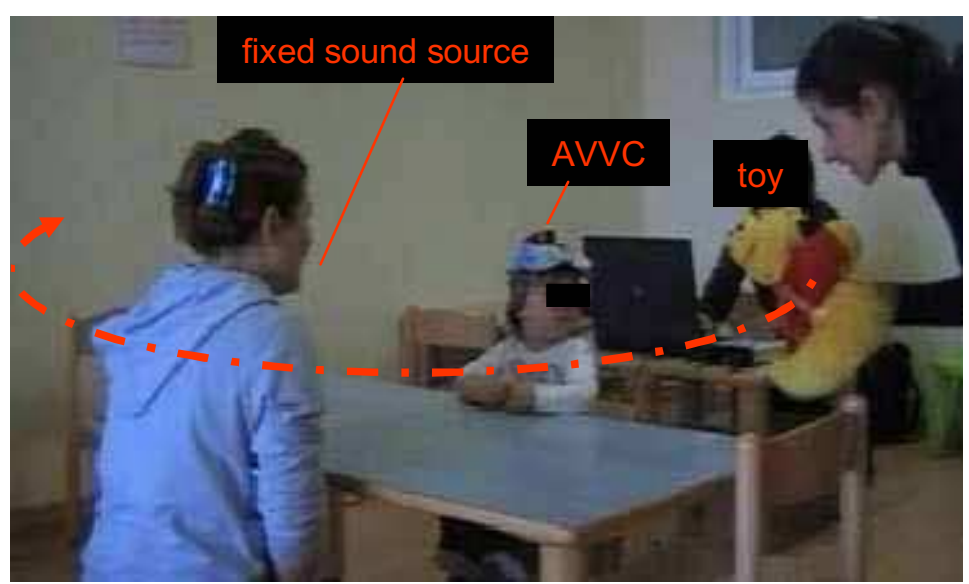

Fig. 13. Ecological calibration protocol for the binaural microphones of the AVVC platform.

An ecological calibration protocol was specifically devised to cope with these issues and considering that children are non-cooperative subjects (especially in the case of children with ASD). As shown in Fig. 13, an experimenter is sitting still in front of the child (already wearing the AVVC with the binaural microphones to be calibrated) and keeps talking, acting thus as a fixed sound source. Another experimenter (not talking) uses a colored toy to capture the child's attention. As the toy is moved around (see dashed line in the), the head of the child, who keeps looking at the toy, spans a wide range of orientations. It is worth noting that the position of the toy is not relevant, its only purpose is inducing the child to orient the head in different directions.

The magneto-inertial device, also mounted on the cap and fixed with respect to the binaural microphones, keeps track of the orientation of the head. In Fig. 14 the head orientation (in degrees) is plotted against the ITD (in milliseconds). The relationship is well fitted to a line $\left(R^{2}=0.98\right)$. The slope of the fitting line is related to the physics of sound localization via ITD cues while the offset of the fitting line is mainly depended on how the cap is fit onto the child's head, which varies from trial to trial.

Clearly, the whole procedure relies on the hypothesis of 'fixed sound source'. Although the experimenter sitting in front of the child is instructed to remain still as much as possible while talking, the child's head is unconstrained and therefore free to translate while orienting towards the moving toy. These factors affect both resolution and accuracy. Nevertheless, preliminary in-field tests, presented in the next section, show how when used in combination with an experimental protocol it can still be used for data segmentation.

\subsubsection{Calibration Procedure for the AVVC eye tracker}

During calibration procedure movements of the pupil in pixels are transformed to eye position in degrees. Eye-tracking systems calibration usually consists of looking at several markers on a screen in order to collect enough data to modify the parameter of an adjustable model, often while keeping the head still. Also, this kind of calibration cannot be easily performed 


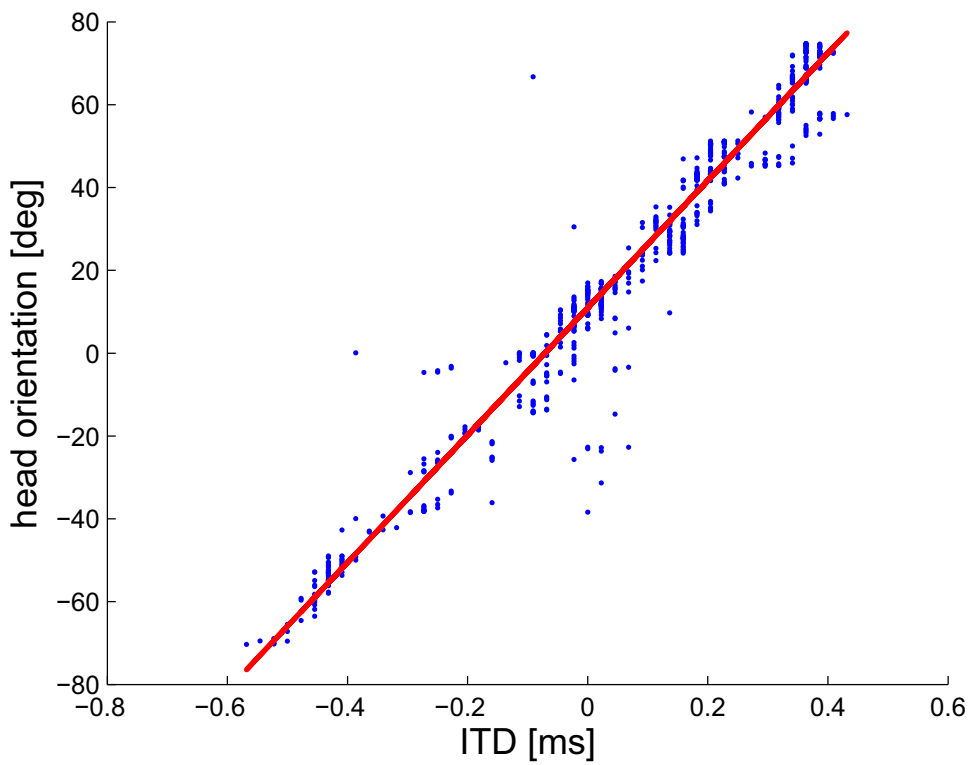

Fig. 14. Calibration curve for the binaural microphones. Fitting values: slope $=150 \mathrm{deg} / \mathrm{ms}$; offset $=11 \mathrm{deg} ; R^{2}=0.98$.

when the users are very young children. We propose a new calibration procedure, inspired by the vestibulo-ocular reflex (VOR) which allows generating compensatory eye movements in response to head motion as sensed by the vestibular organs in the inner ear.

When the head rotates about any axis (horizontal, vertical, or torsional) distant visual images are stabilized by rotating the eyes about the same axis, but in opposite direction (Crawford and Vilis 1991). The gain of the VOR (the ratio of eye angular velocity $\frac{d}{d t} \psi_{e}$ to head angular velocity $\frac{d}{d t} \psi_{h}$ ) is typically around -1 when the eyes are focused on a distant target.

During our calibration procedure, the child is asked to rotate the head to the left and to the right while keeping looking at the caregiver who is sitting in front of him. Head rotation movements are recorded at a frequency of $100 \mathrm{~Hz}$ by the magneto-inertial sensor mounted on the top of the cap. Given the relation between head and eye angular velocities, the head azimuth $\left(\psi_{h}\right)$ correlates with the coordinates ${ }^{2}$ of the pupil in the horizontal plane. Linear fitting can be applied to the calibration curve (see Fig. 15) to extract gain and offset. This allows expressing the eye orientation in degrees rather than in (normalized) pixels, as in Fig. 16

\section{In-Field Testing}

In this section preliminary experimental data relative to in-field testings of both the blockbox and the AVVC platforms are presented. Such tests are significant as they prove usability of the proposed platforms in unstructured environments. In particular, the two platforms

\footnotetext{
${ }^{2}$ Coordinates of the pupil are derived from images and are typically in pixels, i.e. depend on factors such as resolution of the camera and its distance from the pupil. To avoid dealing with zooming factors, distances in pixels are normalized with respect to physiological features such as the width of the eye.
} 


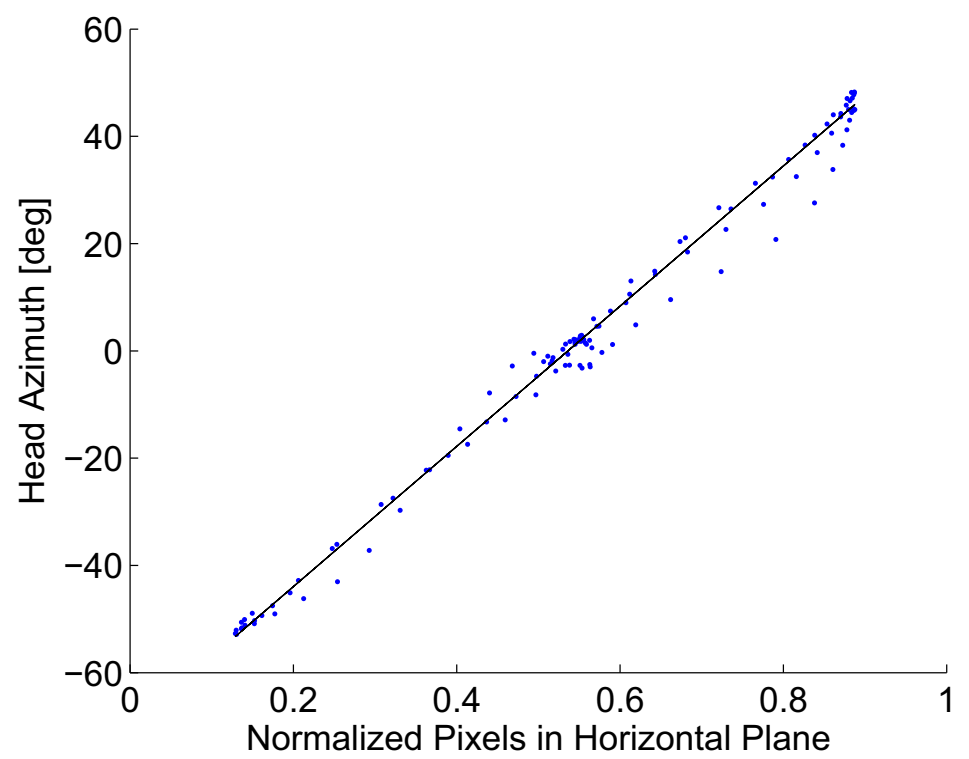

Fig. 15. Head-eye calibration curve: raw data (dots) and fitted line (solid) with $R^{2}>0.90$.
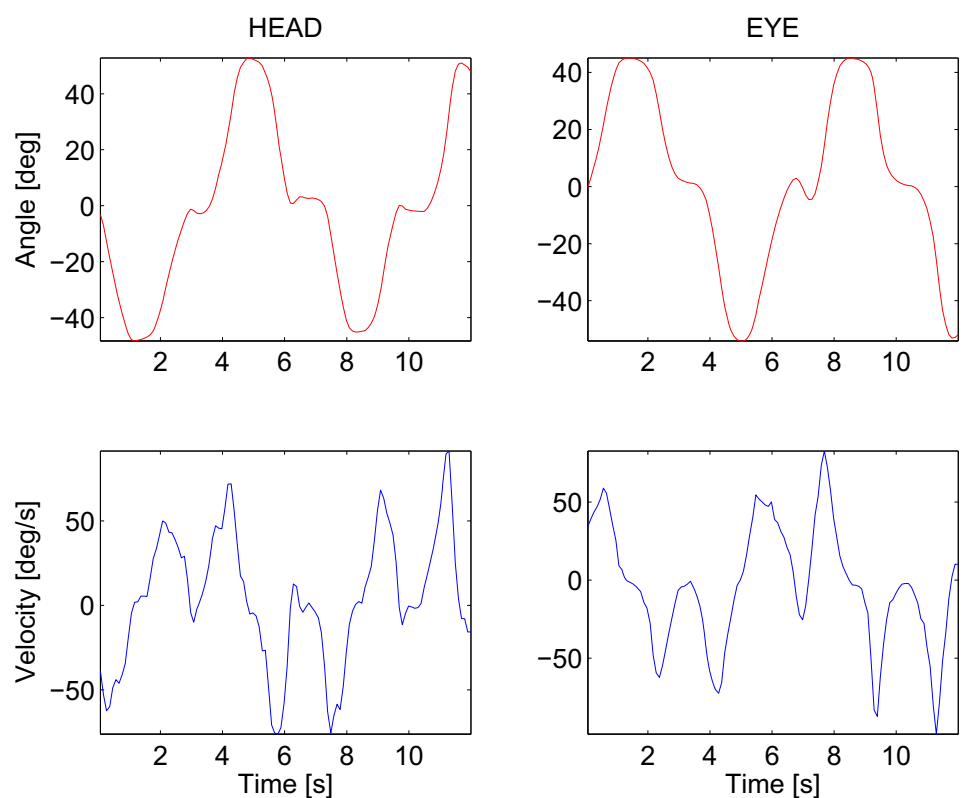

Fig. 16. Head and eye orientation and angular velocity as acquired during a calibration procedure. 
were tested (separately) at our local day-care with typically-developing children aged 12-24 months. The experiments followed the two protocols described in Sec. 3.1 and in Sec. 3.3. Although tested separately, the two platforms could also be used in the same experiment or in conjunction with other devices. In fact, in every experiment, one or more video cameras are also present for recording purposes. A key aspect of multimodal assessment is synchronization among all devices. During the experiments we routinely performed simple actions such as 'tapping' on a switch button in order to trigger contemporaneous physical events, e.g. a flashing light, a buzzing sound, or a touch event. Each sensor present on the scene records the occurrence of at least one such physical triggers (e.g. cameras would capture flashing lights, microphones would capture buzzing sounds, accelerometers would capture 'tapping'). During off-line data pre-processing, the occurrence of such triggering events was then used as a time-stamp to synchronize movie files, with sound files, with kinematic data etc...

\subsection{Experiments with the AVVC platform}

To study sensorimotor coordination during attentional tasks, we need to correlate information coded relatively to different references: the operating space, the head, and the ears. A fixed sound source would appear as moving from the perspective of binaural localization unless the head of the child is held still. Since the child's head cannot be constrained, being able to sense the orientation of the head is crucial.

In particular, with reference to the experiment described in Sec. 3.3, the following relation holds:

$$
\text { sound-in-space }=\text { sound-in-head }+ \text { head-in-space }
$$

where sound-in-head represents the direction of the sound stimulus with respect to the (moving) frame of the subjects's head, sound-in-space is the direction of sound stimulus in the (fixed) frame of the operating space (i.e. the coordinate frame in which the social protocol is planned), and head-in-space is the orientation of the head in the (fixed) frame of the operating space.

Sound-in-head can be estimated via binaural algorithms while head-in-space can be estimated via the inertial-magnetic device, therefore sound-in-space can be reconstructed by adding such two estimates.
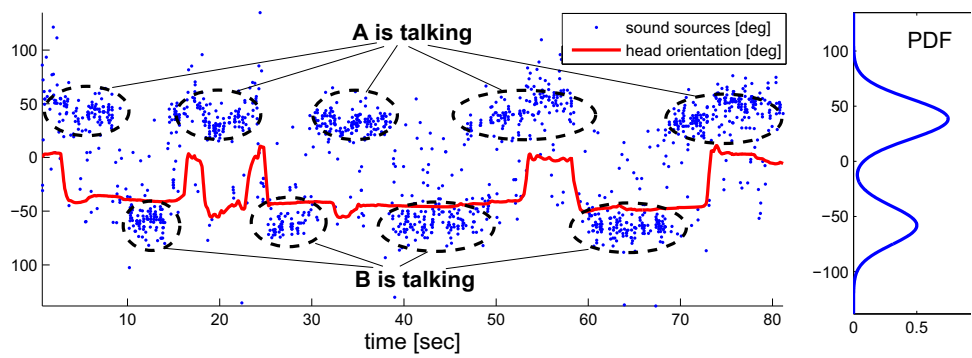

Fig. 17. Plots relative to the AVVC measurements

The data relative to a specific trial are reported in Fig. 17 which plots the head orientation of the child (solid line) as well as the direction of the sound stimuli in the operating space, i.e. sound-in-space (dots). The (fixed) angular position of the two experimenters in Fig. $11 \mathrm{can}$ be identified by the clouds of dots. On the right side of Fig. 17 the distribution (PDF) of such 
dots is shown, the two peaks are relative to the angular position of the experimenters. This information can be used as a spatial filter, i.e. all dots far away from the two peaks can be ignored (despite all the care taken, day-cares remain noisy environments).

From Fig. 17 we can easily tell, at every time, which experimenter is talking and towards whom the child's head is oriented.

Note that the head never fully reaches either of the angular positions of the speakers. The reason is that orienting behaviors also involve eye rotations which should be added to the head orientation. This was confirmed by visual inspection of pictures taken by the eye-cam (e.g. see the details of a child's eyes in Fig. 12). Automatic detection of gaze is still work-inprogress.

\subsection{Experiments with the Block-Box platform}

The block-box prototype described in Sec. 3.1.1 was tested with several typically-developing children at our local day-care. Representative snapshots from one particular trial are shown in Fig. 18 in which the sensorized core was embedded into a cube. In the sequence of snapshots, the child ( 18 months old) first reaches for the cube with his right hand, than adjusts the orientation of cube with both hands and then successfully inserts the cube into the hole, after some final adjustment and pushing.

In the work of Ornkloo and von Hofsten (Ornkloo and von Hofsten 2007), two video cameras monitored the experiment providing respectively a top and a side view. From the videos, after determining the frame during which the object came into contact with the box, both vertical and horizontal alignment of the object with the aperture were evaluated from the specific frame, with a goniometer. Accuracy of the methods highly depends on the quality of the videos. As stated in the paper, the vertical and horizontal alignments were judged by two coders who disagreed on 31 out of 302 cases.

In our experiments, the raw data derived from the inertial-magnetic sensors were first fed into a complementary filter (Campolo et al. 2008) to derive the sequence of orientations of the cube (100 per second, for clarity only few are reported in the middle of Fig 18). Once the orientation of the cube is known, the vertical angular error (i.e. tilt with respect to gravity) and the horizontal angular error (i.e. misalignment between the horizontal projection of the cross-section axes of the object and the axes of the aperture) can be determined at any time, as shown in Fig. 18 (bottom). The time of contact with the box is determined by the peaks of acceleration produced by the shock and distinctively sensed by the accelerometers (2-3 times larger than $g$ ).

In the bottom plot of Fig. 18, the first 4 seconds are relative to the in-air manipulation of the block. Approximately at time $t=4 s$, the first impact with box occurs (detected by the accelerometers), since at this time both errors are below $30 \mathrm{deg}$, the pre-adjustment would be considered correct according to (Ornkloo and von Hofsten 2007). In the remaining 7 seconds, the child tries to insert the cube and only slightly before time $t=11 \mathrm{~s}$ both vertical and horizontal alignment errors drop to zero and the cube can be successfully inserted. As a final note, the exact time of dropping of the cube can also be determined from the accelerometers because for a body in free fall acceleration always drops to zero.

\section{Conclusion}

Although developmental milestones of children are largely described in literature, quantitative normative databases of sensorimotor integration skills in relation to increasingly complex 

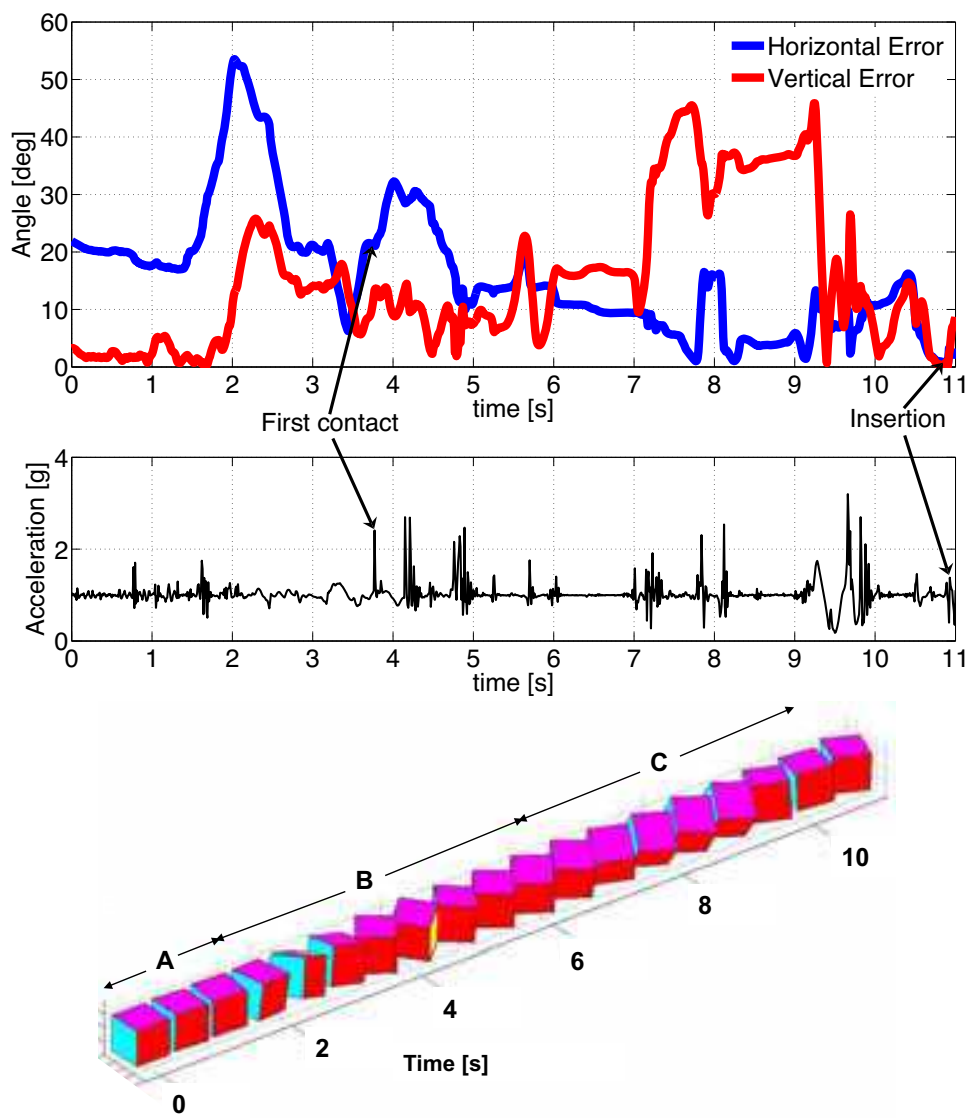

A: Reaching

B: Manipulation
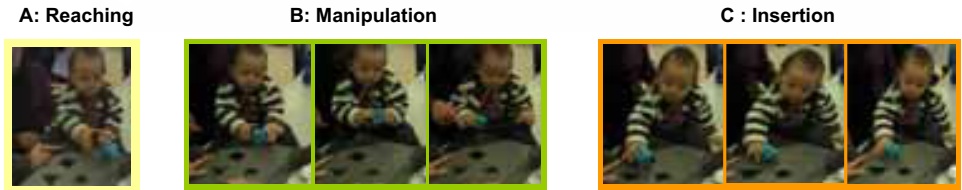

Fig. 18. Experiments with the block-box. Vertical and horizontal alignment errors (top). Reconstructed orientation vs. time (middle). Sequence of snapshots (bottom).

tasks are still lacking. On one hand this would extend the current knowledge on developmental mechanisms, with an impact on Developmental Sciences as well as on Robotics. On the other hand, it would allow early diagnosis of neurodevelopmental disorders such as Autism, with a major impact on society.

For this, technology plays a crucial role. Virtually any toy, tool or piece of garment used by children could host all sorts of technology. Our approach is based on a closed loop dialogue between neuroscientists and bioengineers. The functional specifications for the proposed plat- 
forms are derived from experimental protocols devised by neuroscientists. The selection of the technology strictly followed ecological requirements.

In this paper we present three technological platforms, an instrumented toys, a lightweight bracelet and a wearable cap, specifically devised to assess the development of social attention and spatial cognition in infants.

Deficits in social attention and abnormal social behavior are among the most typical traits of people with Autism. After a child is diagnosed with Autism, i.e. after the 3 years of age, parents retrospectively report failure to use gaze to regulate behavior and to engage in social games of infancy (Landry and Bryson 2004). Assessing social behaviors is per se challenging, due to the multitude of variables to take into account: multi modality is a must.

To this end, we developed the AVVC wearable device aiming at multimodal assessment of gaze and head kinematics in response to sound stimuli. Calibration procedures were also designed which would be appropriate to unstructured environments, appearing as a game to the child. Based on a protocol specifically devised to monitor the response of a child to social stimuli, experiments with the AVVC platform were carried out at a local day-care. These experiments proved that our approach to objective, quantitative and ecological assessment of social behavior is viable. Experimental results also show that, when combined with a structure protocol, semi-automatic segmentation of data is possible.

For spatial cognition, the scientific focus is on the ability of a child to mentally rotate an object in order to fit the appropriate hole. The experimental protocol is devised to assess the vertical and horizontal pre-adjustments of the block (with various levels of difficulty in relation to the different cross-sections) at the time of contact with the box. The 'traditional' methods rely on the (time-consuming) manual scoring of videos, frame-by-frame.

The block-box platform embeds magnetic-inertial sensors. The time of contact can be automatically determined from the large acceleration peaks due to the mechanical shock (i.e. when the block hits the box). For that specific time frame vertical and horizontal alignments are also available via the orientation reconstructed from the raw data (e.g. see values in Fig. 18 for $t=4 s$ ).

In fact, we can reconstruct the orientation at any time. Meaning that pre-adjustment kinematics can be assessed during the whole approaching trajectory. The studies of Mari et al. (Mari et al. 2003) have shown that children with ASD typically have difficulties in activating concurrent motor programs such as reaching for an object and pre- shaping the hand for grasping it. We expect similar findings to hold also for the block-box task, where reaching and pre-adjustment are concurrent motor programs.

In their study, Mari et al., used stereo-photogrammetry, assessing the pre-shaping of the hand via reflective markers on the index finger and the thumb. Although valuable for research, such a method is hardly applicable to clinical practice for screening purposes. The block-box platform is suitable to work in day-cares, or in the office of a pediatrician. In this way a large number of children may actually be objectively monitored.

\section{Acknowledgement}

This work was supported by a grant from the European Union, FP6-NEST/Adventure Programme, contract no. 015636. 


\section{References}

[1] Babcock J. and Pelz J (2004) Building a lightweight eye-tracking headgear, in ACM Eye tracking research and applications symposium, San Antonio, TX, USA, 109-114

[2] Bertone A, Mottron L, Jelenic P, Faubert J. (2003) Motion perception in autism: a "complex" issue, J Cogn Neurosci 15: 218-225.

[3] Blauert J (1997) Spatial Hearing, revised ed. Cambridge, MA: MIT Press

[4] Boddaert N, Chabane N, Belin P, Bourgeois M, Royer V, Barthelemy C, Mouren-Simeoni MC, Philippe A, Brunelle F, Samson Y, Zilbovicius M (2004) Perception of complex sounds in autism: abnormal auditory cortical processing in children. Am J Psychiatry 161:2117-2120.

[5] Byford G.H. (1963) Non-linear relations between the corneo-retinal potential and horizontal eye movements, J. Physiol. (London), 168, 14P-15P

[6] Campolo D, Fabris M, Cavallo G, Accoto D, Keller F, Guglielmelli E (2006) A Novel Procedure for In-field Calibration of Sourceless Inertial/Magnetic Orientation Tracking Wearable Devices, in Proc. of the first IEEE / RAS-EMBS Intl Conf. on Biomedical Robotics and Biomechatronics (BIOROB), pp.471-476, Pisa, Italy, Feb 20-22.

[7] Campolo D, Maini ES, Patane' F, Laschi C, Dario P, Keller F, Guglielmelli E (2007) Design of a Sensorized Ball for Ecological Behavioral Analysis of Infants, IEEE International Conference on Robotics and Automation (ICRA), Pasadena, California, USA, pp. 1318-1323

[8] D. Campolo, L. Schenato, L. Pi, X. Deng, E. Guglielmelli (2009) Attitude Estimation of a Biologically Inspired Robotic Housefly via Multimodal Sensor Fusion, RSJ Advanced Robotics Journal 23:955-977

[9] Cornsweet T. N. and Crane H.D. (1973) Accurate two-dimensional eye tracker using first and fourth Purkinje images, J. Opt. Sc. Am., 63, 921-928

[10] Courchesne E, Kilman BA, Galambos R, Lincoln AJ (1984) Autism: processing of novel auditory information assessed by event- related brain potentials. Electroencephalogr Clin Neurophysiol 59:238-248.

[11] Crawford JD, Vilis T (1991) Axes of eye rotation and Listing's law during rotations of the head. Journal of Neurophysiology, 65(3), 407-423

[12] Dawson G, Toth K, Abbott R, Osterling J, Munson J, Estes A, Liaw J (2004) Early Social Attention Impairments in Autism: Social Orienting, Joint Attention, and Attention to Distress, Developmental Psychology, 40:271-283

[13] DiScenna A. O., Das V., Zivotofsky A. Z., Seidman S. H., Leigh R. J.(1995) Evaluation of a video tracking device for measurement of horizontal and vertical eye rotations during locomotion, Journal of Neuroscience Methods, 58, 89-94

[14] Einspieler C. , Prechtl H. F. R. (2005), PrechtlŠs Assessment of General Movements: A Diagnostic Tool for the Functional Assessment of the Young Nervous System, Mental Retardation and Developmental Disabilities Research Reviews, 11:61-67.

[15] Eizenman M., Frecker R. C., and Hallet P.E. (1984) Precise non-contacting measurement of eye movements using corneal reflex, Vis. Res., 24:167-174

[16] Gerlai R (2002) Phenomics: fiction or the future?, in Trends Neurosci., 25:506-9

[17] Grelotti DJ, Gauthier I, Schultz RT (2003) Social interest and the development of cortical face specialization: what autism teaches us about face processing. Dev Psychobiol 40:213225.

[18] Hallett M, Lebiedowska MK, Thomas SL, Stanhope SJ, Denckla MB, Rumsey J (1993) Locomotion of autistic adults. Arch Neurol 50:1304-1308 
[19] Haslwanter T. (1995) Mathematics of three-dimensional eye rotations, Vision Res., 42, 1053-1061

[20] Hobson RP, Bishop M (2003) The pathogenesis of autism: insights from congenital blindness. Philos Trans R Soc Lond B Biol Sci., 358:335-344.

[21] Jovanov E., Milenkovic A., Otto C., de Groen P. C. (2005), A wireless body area network of intelligent motion sensors for computer assisted physical rehabilitation, J. Neuroengineering Rehabil., 2:6 doi:10.1186/1743-0003-2-6.

[22] Keller F, Persico AM (2003) The neurobiological context of autism, Mol. Neurobiol.. 28:1-22

[23] Kemp B, Janssen AJMW, van der Kamp B (1998) Body position can be monitored in 3D using miniature accelerometers and earth-magnetic field sensors, Electroencephalography and Clinical Neurophysiology, 109:484-488

[24] Landau U. M. (1987) Estimation of a circular arc centre and its radius, Comput. Vis Graph. Image Process., 38:317-326

[25] Landry R, Bryson SE (2004) Impaired disengagement of attention in young children with autism, J Child Psychol Psychiatry, 45: 1115-22.

[26] Lotters JC, Schipper J, Veltink PH, Olthuis W, Bergveld P (1998) Procedure for in-use calibration of triaxial accelerometers in medical applications, Sensors and Actuators A, 68:221-228

[27] Maestro S, Muratori F, Cavallaro MC, Pei F, Stern D, Golse B, Palacio-Espasa F (2002) Attentional skills during the first 6 months of age in autism spectrum disorder. $J$ Am Acad Child Adolesc Psychiatry, 41:1239-1245.

[28] Mari M, Castiello U, Marks D, Marraffa C, Prior M (2003) The reach-to-grasp movement in children with autism spectrum disorder, Philos Trans R Soc Lond B Biol Sci., 358:393-403

[29] Metta G, Sandini G, Konczak J (1999) A developmental approach to visually-guided reaching in artificial systems, Neural Networks, 12:1413-1427

[30] Milne E, Swettenham J, Hansen P, Campbell R, Jeffries H, Plaisted K (2002) High motion coherence thresholds in children with autism. J Child Psychol Psychiatry 43:255-263.

[31] Minshew NJ, Sung K, Jones BL, Furman JM (2004) Underdevelopment of the postural control system in autism, Neurology, 63:2056-2061

[32] Molloy CA, Dietrich KN, Bhattacharya A (2003) Postural stability in children with autism spectrum disorder, J Autism Dev Disord, 33:643-652

[33] Mundy P, Neal R (2001) Neural plasticity, joint attention and a transactional socialorienting model of autism. In L. Glidden (Ed.), International review of research in mental reterdation, Vol. 23, Autism (pp. 139-168), New York: Accademic Press

[34] Ornkloo H, von Hofsten C (2007) Fitting objects into holes: on the development of spatial cognition skills, Dev Psychol., 43:404-16

[35] Pelz J., Canosa R., Babcock J., Kucharczyk D., Silver A., and Konno D. (2000) Portable eyetracking: Astudy of eye movements, in Proceedings of SPIE, Human Vision and Electronic Imaging, San Jose, CA, USA, 566-582

[36] Pierce K, Courchesne E (2001) Evidence for a cerebellar role in reduced exploration and stereotyped behavior in autism, Biol Psychiatry, 49:655-664

[37] Prechtl H. F. R. General (2001), Movement assessment as a method of developmental neurology: new paradigms and their consequences, Developmental Medicine $\mathcal{E}$ Child Neurology, 43: 836-842. 
[38] Rinehart NJ, Bradshaw JL, Brereton AV, Tonge BJ (2001) Movement preparation in highfunctioning autism and Asperger disorder: a serial choice reaction time task involving motor reprogramming, J Autism Dev Disord, 31:79-88

[39] Robinson D. A. (1963) A method for measuring eye movement using a scleral search coil in a magnetic field, IEEE Trans. Biom. Eng., 10, 137-145

[40] Rucci M, Wray J (1999) Binaural cross-correlation and auditory localization in barn owl: a theoretical study, Neural Networks, 12:31-42

[41] Schmitz C, Martineau J, Barthelemy C, Assaiante C (2003) Motor control and children with autism: deficit of anticipatory function?, Neurosci. Lett., 348:17-20

[42] Spencer J, O’Brien J, Riggs K, Braddick O, Atkinson J, Wattam- Bell J (2000) Motion processing in autism: evidence for a dorsal stream deficiency, Neuroreport, 11:2765-2767.

[43] Sweeney JA, Takarae Y, Macmillan C, Luna B, Minshew NJ (2004) Eye movements in neurodevelopmental disorders, Curr Opin Neurol., 17:37-42

[44] Swettenham J, Baron-Cohen S, Charman T, Cox A, Baird G, Drew A, Rees L, Wheelwright $S$ (1998) The frequency and distribution of spontaneous attention shifts between social and nonsocial stimuli in autistic, typically developing, and nonautistic developmentally delayed infants. J Child Psychol Psychiatry 39:747-753.

[45] Takarae Y, Minshew NJ, Luna B, Krisky CM, Sweeney JA (2004) Pursuit eye movement deficits in autism, Brain 127:2584-2594

[46] Taffoni F, Campolo D, Delafield-Butt J, Keller F, Guglielmelli E, (2008) Design and assembling of a magneto-inertial wearable device for ecological behavioral analysis of infants, IEEE/RSJ International Conference on Intelligent Robots and Systems (IROS), Nice, France, pp. 3832-3837.

[47] Teitelbaum P, Teitelbaum O, Nye J, Fryman J, and Maurer RG (1998) Movement analysis in infancy may be useful for early diagnosis of autism, Proc Natl Acad Sci USA, 95: 1398213987

[48] Teitelbaum O, Benton T, Shah PK, Prince A, Kelly JL, Teitelbaum P (2004) EshkolWachman movement notation in diagnosis: the early detection of Asperger's syndrome, Proc Natl Acad Sci USA 101:11909-11914

[49] Trevarthen C, Daniel S, (2005) Disorganized rhythm and synchrony: Early signs of autism and Rett syndrome, Brain and Development, 27:25-34

[50] Young L., Sheena D. (1975) Survey of eye movement recoding methods. Behavior research methods $\mathcal{E}$ instrumentation, 7(5), 397-429

[51] Welch G, Foxlin E (2002) Motion Tracking: No Silver Bullet, but a Respectable Arsenal, IEEE Computer Graphics and Applications, 22:24-38 


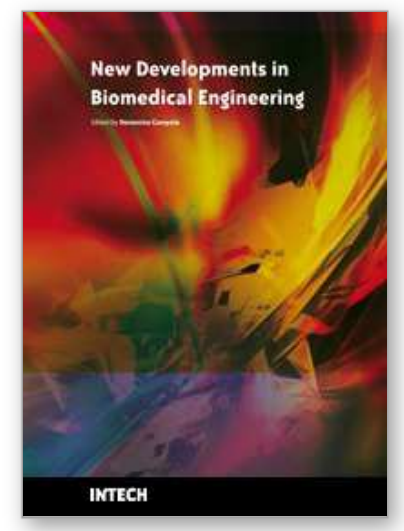

\author{
New Developments in Biomedical Engineering \\ Edited by Domenico Campolo
}

ISBN 978-953-7619-57-2

Hard cover, 714 pages

Publisher InTech

Published online 01, January, 2010

Published in print edition January, 2010

Biomedical Engineering is a highly interdisciplinary and well established discipline spanning across engineering, medicine and biology. A single definition of Biomedical Engineering is hardly unanimously accepted but it is often easier to identify what activities are included in it. This volume collects works on recent advances in Biomedical Engineering and provides a bird-view on a very broad field, ranging from purely theoretical frameworks to clinical applications and from diagnosis to treatment.

\title{
How to reference
}

In order to correctly reference this scholarly work, feel free to copy and paste the following:

Domenico Campolo, Fabrizio Taffoni, Giuseppina Schiavone, Domenico Formica, Eugenio Guglielmelli and Flavio Keller (2010). Neuro-Developmental Engineering: towards Early Diagnosis of Neuro-Developmental Disorders, New Developments in Biomedical Engineering, Domenico Campolo (Ed.), ISBN: 978-953-7619-572, InTech, Available from: http://www.intechopen.com/books/new-developments-in-biomedicalengineering/neuro-developmental-engineering-towards-early-diagnosis-of-neuro-developmental-disorders

\section{INTECH}

open science | open minds

\section{InTech Europe}

University Campus STeP Ri

Slavka Krautzeka 83/A

51000 Rijeka, Croatia

Phone: +385 (51) 770447

Fax: +385 (51) 686166

www.intechopen.com

\section{InTech China}

Unit 405, Office Block, Hotel Equatorial Shanghai No.65, Yan An Road (West), Shanghai, 200040, China 中国上海市延安西路65号上海国际贵都大饭店办公楼405单元 Phone: +86-21-62489820

Fax: $+86-21-62489821$ 
(C) 2010 The Author(s). Licensee IntechOpen. This chapter is distributed under the terms of the Creative Commons Attribution-NonCommercial-ShareAlike-3.0 License, which permits use, distribution and reproduction for non-commercial purposes, provided the original is properly cited and derivative works building on this content are distributed under the same license. 